\title{
Viscosity solutions of systems of variational inequalities with interconnected bilateral obstacles of non-local type.
}

\author{
Said Hamadène* and Xuzhe Zhao ${ }^{\dagger}$
}

October 18, 2018

\begin{abstract}
In this paper, we study systems of nonlinear second-order variational inequalities with interconnected bilateral obstacles with non-local terms. They are of min-max and max-min types and related to a multiple modes zero-sum switching game in the jump-diffusion model. Using systems of penalized reflected backward SDEs with jumps and unilateral interconnected obstacles, and their associated deterministic functions, we construct for each system a continuous viscosity solution which is unique in the class of functions with polynomial growth.
\end{abstract}

Keywords: Switching zero-sum games ; non-local variational inequalities ; backward stochastic differential equation ; Hamilton-Jacobi-Bellman-Isaacs equation ; Perron's method ; viscosity solution.

AMS subject classification: 49N70, 49L25, 90C39, 93E20.

\section{Introduction}

During the last decade optimal stochastic switching problems have attracted a lot of research activity (see e.g. $[6,11,13,12,16,17,18,19,22,26,30]$ and the references therein) in connection with their various applications especially in the economic and finance spheres, such as energy, etc. Comparatively, switching games, of zero-sum or nonzero-sum types, have been less considered even though there are some works in this field including $[10,23,24,25,28]$. In these latter articles, the Hamilton-Jacobi-Bellmans-Isaacs PDE, which is of min-max or max-min type, associated with the zero-sum switching stochastic game is studied from the point of view of viscosity solution theory. The probabilistic version of those works is considered in $[10,23]$ where it is shown that the BSDE system associated with the zero-sum game has a solution. In [10], uniqueness of the solution, which is an involved question, is proved as well. The issue of existence of a value or a saddle-point for the game is also addressed in [10], where it is shown that the game has a saddle-point

\footnotetext{
*Université du Maine, LMM, Avenue Olivier Messiaen, 72085 Le Mans, Cedex 9, France. Email: hamadene@univ-lemans.fr

$\dagger$ Applied Mathematics Department School of Finance University of Foreign Studies, Guangzhou 510420, P.R.China. E-mail: sosmall129@hotmail.com
} 
when the switching costs and utilities are decoupled. This existence is deeply related to the optimal policy of a standard optimal switching problem. The general case still open.

Except articles [20,16], all the previous works deal with the case of Brownian noise. In [20], the framework where the noise is driven by a Lévy process is studied in detail. The main motivation is that models which include Poisson jumps have the feature to be more realistic since they capture non-predictable events, e.g. in the energy market, jumps of the prices due to sudden weather changes, etc. Therefore the main objective of this work is the extension to the model with jumps of the paper [10], where the authors have studied systems of variational inequalities with interconnected lower and upper obstacles, which arise as the Hamilton-JacobiBellman-Isaacs equation in a multiple modes switching game between two players in the framework without jumps. Precisely we consider the following system of non-local variational inequalities or integral-partial differential equations (IPDEs for short): For every pair $(i, j)$ in the finite set of modes $A^{1} \times A^{2}$,

$$
\left\{\begin{array}{l}
\min \left\{\left(v^{i j}-L^{i j}\left[\left(v^{k l}\right)_{\left.\left.(k, l) \in A^{1} \times A^{2}\right]\right)(t, x), \max \left\{\left(v^{i j}-U^{i j}\left[\left(v^{k l}\right)_{\left.\left.(k, l) \in A^{1} \times A^{2}\right]\right)}(t, x),\right.\right.\right.}\right.\right.\right. \\
-\partial_{t} v^{i j}(t, x)-\mathcal{L} v^{i j}(t, x)-g^{i j}\left(t, x,\left(v^{k l}(t, x)\right)_{\left.\left.\left.(k, l) \in A^{1} \times A^{2}, \sigma(t, x)^{\top} D_{x} v^{i j}(t, x), I_{i j}\left(t, x, v^{i j}\right)\right)\right\}\right\}=0 ;} v^{i j}(T, x)=h^{i j}(x)\right.
\end{array}\right.
$$

where, for any $(t, x) \in[0, T] \times \mathbf{R}^{k}$,

$$
\begin{aligned}
& \text { a) } \mathcal{L} \phi(t, x):=b(t, x)^{\top} D_{x} \phi(t, x)+\frac{1}{2} \operatorname{Tr}\left[\sigma \sigma^{\top}(t, x) D_{x x}^{2} \phi(t, x)\right]+ \\
& \int_{E}\left(\phi(t, x+\beta(x, e))-\phi(t, x)-D_{x} \phi(t, x) \beta(x, e)\right) n(d e) ; \\
& \text { b) } I_{i j}(t, x, \phi)=\int_{E}(\phi(t, x+\beta(x, e))-\phi(t, x)) \gamma^{i j}(x, e) n(d e) \text {; } \\
& \text { c) } \left.L^{i j}\left[\left(v^{k l}\right)_{\left.(k, l) \in A^{1} \times A^{2}\right]}\right](t, x):=\max _{k \neq i}\left(v^{k j}-\underline{g}_{i k}\right)(t, x)\right) \text { and } \\
& \left.U^{i j}\left[\left(v^{k l}\right)_{\left.(k, l) \in A^{1} \times A^{2}\right]}\right](t, x):=\min _{l \neq j}\left(v^{i l}+\bar{g}_{j l}\right)(t, x)\right) .
\end{aligned}
$$

The function $\underline{g}_{i k}$ (resp. $\bar{g}_{j l}$ ) stands for the switching cost of the maximizer (resp. minimizer) when she makes the decision to switch from mode $i$ to mode $k$ (resp. mode $j$ to mode $l$ ) while the function $g^{i j}$ (resp. $h^{i j}$ ) is the instantaneous (resp. terminal) payoff when the maximizer (resp. minimizer) chooses mode $i$ (resp. $j$ ). The non-local terms which appear in (1.1) and given in a), b) above stem from the jumps of the dynamics of the system which is of jump-diffusion type (see (2.5) below). Finally note that the obstacles in (1.1) depend on the solution.

In this paper we show that system (1.1) has a continuous solution in viscosity sense which is moreover unique in the class of functions which have polynomial growth. As a by-product we obtain the same conclusion for the max-min system (4.26). Our work should be seen as a starting point for future research in this field (e.g. improvement of the results, numerics, etc.).

This paper is organized as follows: In Section 2, we fix the notations, assumptions, definitions and set up accurately the problem. In Section 3, we prove a comparison result between the subsolutions and supersolutions of system (1.1) when they have polynomial growth. As an immediate consequence, the solutions of (1.1) with polynomial growth is necessarily continuous and unique. In Section 4, we introduce 
systems of integral-partial differential equations with lower (resp. upper) interconnected obstacles which are obtained by the penalization of the upper (resp. lower) obstacles of system (1.1). They are approximating schemes for (1.1) and max-min system respectively. We highlight some of their properties in making the connection with systems of reflected BSDEs with lower (resp. upper) obstacles. Later on we show that system (1.1) has a subsolution and a supersolution as well. Finally by Perron's method we show that it has a unique solution. As a by product we show also that the max-min system has a unique solution. At the end, there is an Appendix, where we give another definition of the viscosity solution of system (1.1) which uses "local" maxima and minima and which is inspired by the work by Barles-Imbert in [4].

\section{Preliminaries}

Let $\left(\Omega, \mathcal{F},\left(\mathcal{F}_{t}\right)_{t \geq 0}, P\right)$ be a stochastic basis such that $\mathcal{F}_{0}$ contains all $P$-null sets of $\mathcal{F}$, and $\mathcal{F}_{t^{+}}=\bigcap_{\varepsilon>0} \mathcal{F}_{t+\varepsilon}:=$ $\mathcal{F}_{t}, t \geq 0$. We suppose that the filtration is generated by the following two mutually independent processes: - a $d$-dimensional standard Brownian motion $\left(B_{t}\right)_{t \geq 0}$

- a Poisson random measure $N$ on $\mathbf{R}_{+} \times E$, where $E=\mathbf{R}^{l}-\{0\}$ ( $l \geq 1$ fixed) is equipped with its Borel field $\mathcal{B}_{E}$, with compensator $\nu(d t, d e)=d t n(d e)$, such that $\{\hat{N}((0, t] \times A)=(N-\nu)((0, t] \times A)\}_{0 \leq t \leq T}$ is an $\mathcal{F}_{t}$-martingale for all $A \in \mathcal{B}_{E}$ satisfying $n(A)<\infty$. The measure $n$ is assumed to be $\sigma$-finite on $\left(E, \mathcal{B}_{E}\right)$ and integrates $\left(1 \wedge|e|^{2}\right)_{e \in E}$.

Let $T$ be a fixed positive constant and let $A^{1}$ (resp. $A^{2}$ ) denote the set of switching modes for player 1 (resp. player 2) whose cardinal is $m_{1}$ (resp. $m_{2}$ ). The set $A^{1} \times A^{2}$ will be sometimes simply denoted by $\Gamma$. For $(i, j) \in \Gamma$, we set $A_{i}^{1}:=A^{1}-\{i\}, A_{j}^{2}:=A^{2}-\{j\}$ and $\Gamma^{-(i, j)}=\Gamma-\{(i, j)\}$. Next, for $\vec{y}=\left(y^{k l}\right)_{(k, l) \in A^{1} \times A^{2}} \in \mathbf{R}^{m_{1} \times m_{2}}$ and $y_{1} \in \mathbf{R}$, we denote by $\left[\vec{y}^{i j}, y_{1}\right]$ the matrix obtained from $\vec{y}$ by replacing the element $y^{i j}$ with $y_{1}$.

A function $\Phi:(t, x) \in[0, T] \times \mathbf{R}^{k} \mapsto \Phi(t, x) \in \mathbf{R}$ is called of polynomial growth if there exist two non-negative real constant $C$ and $\gamma$ such that for any $(t, x) \in[0, T] \times \mathbf{R}^{k}$,

$$
|\Phi(t, x)| \leq C\left(1+|x|^{\gamma}\right)
$$

Hereafter, this class of functions is denoted by $\Pi_{g}$.

We now define the probabilistic tools and sets we need later. Let:

(i) $\mathcal{P}$ be the $\sigma$-algebra of $\mathcal{F}_{t}$-predictable subsets of $\Omega \times[0, T]$;

(ii) $\mathcal{H}^{2}:=\left\{\varphi:=\left(\varphi_{t}\right)_{t \leq T}\right.$ is an $\mathbf{R}^{d}$-valued, $\mathcal{F}_{t}$-progressively measurable process s.t. $\|\varphi\|_{\mathcal{H}^{2}}^{2}:=\mathbb{E}\left(\int_{0}^{T}\left|\varphi_{t}\right|^{2} d t\right)<$ $\infty\}$;

(iii) $\mathcal{S}^{2}:=\left\{\xi:=\left(\xi_{t}\right)_{t \leq T}\right.$ is an $\mathbf{R}$-valued, $\mathcal{F}_{t^{-}}$-adapted RCLL process s.t. $\left.\|\xi\|_{\mathcal{S}^{2}}^{2}:=\mathbb{E}\left[\sup _{0 \leq t \leq T}\left|\xi_{t}\right|^{2}\right]<\infty\right\}$; $\mathcal{A}^{2}$ is the subspace of $\mathcal{S}^{2}$ of continuous non-decreasing processes null at $t=0$;

(iv) $\mathcal{H}^{2}(\tilde{N}):=\left\{U: \Omega \times[0, T] \times E \rightarrow \mathbf{R}, \mathcal{P} \otimes \mathcal{B}_{E^{-}}\right.$measurable and s.t. $\|U\|_{\mathcal{H}^{2}(\tilde{N})}^{2}:=\mathbb{E}\left(\int_{0}^{T} \int_{E}\left|U_{t}(e)\right|^{2} n(d e) d t\right)<$ $\infty\}$. 
The main objective of this paper is to investigate the problem of existence and uniqueness of a viscosity solutions $\vec{v}(t, x):=\left(v^{i j}(t, x)\right)_{(i, j) \in A^{1} \times A^{2}}$ of the following system of non-local variational inequalities (SVI in short) or IPDEs with upper and lower interconnected obstacles: $\forall(i, j) \in A^{1} \times A^{2}$,

$$
\left\{\begin{array}{l}
\min \left\{\left(v^{i j}-L^{i j}[\vec{v}]\right)(t, x) ; \max \left\{\left(v^{i j}-U^{i j}[\vec{v}]\right)(t, x) ;\right.\right. \\
\left.\left.-\partial_{t} v^{i j}(t, x)-\mathcal{L} v^{i j}(t, x)-g^{i j}\left(t, x,\left(v^{k l}(t, x)\right)_{(k, l) \in A^{1} \times A^{2}}, \sigma(t, x)^{\top} D_{x} v^{i j}(t, x), I_{i j}\left(t, x, v^{i j}\right)\right)\right\}\right\}=0 ; \\
v^{i j}(T, x)=h^{i j}(x),
\end{array}\right.
$$

where for any $(i, j) \in \Gamma,(t, x) \in[0, T] \times \mathbf{R}^{k}$ and $\phi \in \mathcal{C}^{1,2}, L^{i j}[\vec{v}], U^{i j}[\vec{v}], \mathcal{L} v^{i j}(t, x)$ and $I_{i j}(t, x, \phi)$ are given in (1.2). The functions $\underline{g}_{i k}, \bar{g}_{i k}, \beta$ and $\gamma^{i j}$ are given and will be specified more later.

Next for $\delta>0,(t, x) \in[0, T] \times \mathbf{R}^{k}, \zeta \in \mathbf{R}^{k}, \phi$ a $\mathcal{C}^{1,2}$-function and $(i, j) \in \Gamma$, let us set:

$$
\begin{aligned}
& \text { (a) } I_{\delta}^{1}(t, x, \phi)=\int_{|e| \leq \delta}\left(\phi(t, x+\beta(x, e))-\phi(t, x)-D_{x} \phi(t, x) \beta(x, e)\right) n(d e) ; \\
& (b) I_{\delta}^{2}(t, x, \zeta, \phi)=\int_{|e| \geq \delta}(\phi(t, x+\beta(x, e))-\phi(t, x)-\zeta \beta(x, e)) n(d e) ; \\
& (c) I_{i j}^{1, \delta}(t, x, \phi)=\int_{|e| \leq \delta}(\phi(t, x+\beta(x, e))-\phi(t, x)) \gamma^{i j}(x, e) n(d e) ; \\
& (d) I_{i j}^{2, \delta}(t, x, \phi)=\int_{|e| \geq \delta}(\phi(t, x+\beta(x, e))-\phi(t, x)) \gamma^{i j}(x, e) n(d e) ; \\
& \text { (e) } \overline{\mathcal{L}} \phi(t, x):=b(t, x)^{\top} D_{x} \phi(t, x)+\frac{1}{2} \operatorname{Tr}\left[\sigma \sigma^{\top}(t, x) D_{x x}^{2} \phi(t, x)\right] .
\end{aligned}
$$

Note that for any $\delta>0$ and $(i, j) \in A^{1} \times A^{2}$,

$$
I(t, x, \phi)=I_{\delta}^{1}(t, x, \phi)+I_{\delta}^{2}\left(t, x, D_{x} \phi, \phi\right) \text { and } I_{i j}(t, x, \phi)=I_{i j}^{1, \delta}(t, x, \phi)+I_{i j}^{2, \delta}(t, x, \phi) .
$$

Next the following assumptions will be in force throughout the rest of this paper.

$\underline{(\mathrm{A} 0)}$ :

(i) The function $b(t, x)$ (resp. $\sigma(t, x)$ ): $[0, T] \times \mathbf{R}^{k} \rightarrow \mathbf{R}^{k}$ (resp. $\mathbf{R}^{k \times d}$ ) is jointly continuous in $(t, x$ ) and Lipschitz continuous w.r.t. $x$, meaning that there exists a non-negative constant $C$ such that for any $\left(t, x, x^{\prime}\right) \in[0, T] \times \mathbf{R}^{k+k}$ we have:

$$
\left|\sigma(t, x)-\sigma\left(t, x^{\prime}\right)\right|+\left|b(t, x)-b\left(t, x^{\prime}\right)\right| \leq C\left|x-x^{\prime}\right| .
$$

Combining this property with continuity one deduces that $b$ and $\sigma$ are of linear growth w.r.t. $x$, i.e.,

$$
|b(t, x)|+|\sigma(t, x)| \leq C(1+|x|) .
$$

(ii) The function $\beta: \mathbf{R}^{k} \times E \rightarrow \mathbf{R}^{k}$ is measurable, and such that for some real $K$,

$$
|\beta(x, e)| \leq K(1 \wedge|e|) \text { and }\left|\beta(x, e)-\beta\left(x^{\prime}, e\right)\right| \leq K\left|x-x^{\prime}\right|(1 \wedge|e|), \forall e \in E \text { and } x, x^{\prime} \in \mathbf{R}^{k} .
$$

\section{$\underline{(\mathrm{A} 1)}$ :}

For any $(i, j) \in \Gamma$, the function $g^{i j}:(t, x, \vec{y}, z, q) \in[0, T] \times \mathbf{R}^{k+m_{1} \times m_{2}+d+1} \longmapsto g^{i j}(t, x, \vec{y}, z, q) \in \mathbf{R}$ verifies:

(i) it is continuous in $(t, x)$ uniformly w.r.t. the other variables $(\vec{y}, z, q)$ and for any $(t, x)$ the mapping 
$(t, x) \mapsto g^{i j}(t, x, 0,0,0)$ is of polynomial growth ;

(ii) it satisfies the standard hypothesis of Lipschitz continuity w.r.t. the variables $(\vec{y}, z, q)$, i.e. for any $(t, x) \in[0, T] \times \mathbf{R}^{k},\left(\vec{y}_{1}, \vec{y}_{2}\right) \in\left(\mathbf{R}^{m_{1} \times m_{2}}\right)^{2}$ and $\left(z_{1}, z_{2}\right) \in\left(\mathbf{R}^{d}\right)^{2}, q_{1}, q_{2} \in \mathbf{R}$, it holds

$$
\left|g^{i j}\left(t, x, \vec{y}_{1}, z_{1}, q_{1}\right)-g^{i j}\left(t, x, \vec{y}_{2}, z_{2}, q_{2}\right)\right| \leq C\left(\left|\vec{y}_{1}-\vec{y}_{2}\right|+\left|z_{1}-z_{2}\right|+\left|q_{1}-q_{2}\right|\right)
$$

where $|\vec{y}|$ stands for the standard Euclidean norm of $\vec{y}$ in $\mathbf{R}^{m_{1} \times m_{2}}$;

(iii) the mapping $q \mapsto g^{i j}(t, x, y, z, q)$ is non-decreasing, for all fixed $(t, x, y, z) \in[0, T] \times \mathbf{R}^{k+m_{1} \times m_{2}+d}$.

Next for any $(i, j) \in \Gamma$, the function $\gamma^{i j}: \mathbf{R}^{k} \times E \rightarrow \mathbf{R}$ verifies for some constant $C$ :

(a) $\left|\gamma^{i j}(x, e)-\gamma^{i j}\left(x^{\prime}, e\right)\right| \leq C\left|x-x^{\prime}\right|(1 \wedge|e|), \quad x, x^{\prime} \in \mathbf{R}^{k}$ and $e \in E$;

(b) $0 \leq \gamma^{i j}(x, e) \leq C(1 \wedge|e|), \quad x \in \mathbf{R}^{k}$ and $e \in E$.

Finally let us define functions $\left(f^{i j}\right)_{(i, j) \in A^{1} \times A^{2}}$, on $[0, T] \times \mathbf{R}^{k+m_{1} \times m_{2}+d} \times \mathcal{L}_{\mathbf{R}}^{2}\left(E, \mathcal{B}_{E}, n\right)$, as follows:

$$
f^{i j}(t, x, \vec{y}, z, u):=g^{i j}\left(t, x, \vec{y}, z, \int_{E} u(e) \gamma^{i j}(x, e) n(d e)\right) .
$$

(A2): Monotonicity: For any $(i, j) \in \Gamma$ and any $(k, l) \neq(i, j)$, the mapping $y^{k l} \rightarrow g^{i j}(t, x, \vec{y}, z, u)$ is nondecreasing.

(A3): The non free loop property: The switching costs $\underline{g}_{i k}$ and $\bar{g}_{j l}$ are non-negative, jointly continuous in $(t, x)$, belong to $\Pi_{g}$ and satisfy the following condition: For any loop in $A^{1} \times A^{2}$, i.e., any sequence of pairs $\left(i_{1}, j_{1}\right), \ldots,\left(i_{N}, j_{N}\right)$ of $A^{1} \times A^{2} \operatorname{such}$ that $\left(i_{N}, j_{N}\right)=\left(i_{1}, j_{1}\right), \operatorname{card}\left\{\left(i_{1}, j_{1}\right), \ldots,\left(i_{N}, j_{N}\right)\right\}=N-1$ and $\forall q=1, \ldots, N-1$, either $i_{q+1}=i_{q}$ or $j_{q+1}=j_{q}$, we have:

$$
\forall(t, x) \in[0, T] \times \mathbf{R}^{k}, \quad \sum_{q=1, N-1} \varphi_{i_{q} j_{q}}(t, x) \neq 0,
$$

where for any $q=1, \ldots, N-1, \varphi_{i_{q} j_{q}}(t, x)=-\underline{g}_{i_{q} i_{q+1}}(t, x) \mathbb{1}_{i_{q} \neq i_{q+1}}+\bar{g}_{j_{q} j_{q+1}}(t, x) \mathbb{1}_{j_{q} \neq j_{q+1}}$.

(A4): The functions $h^{i j}: \mathbf{R}^{k} \rightarrow \mathbf{R}$ are continuous w.r.t. $x$, belong to class $\Pi_{g}$ and satisfy:

$$
\forall(i, j) \in \Gamma, x \in \mathbf{R}^{k}, \max _{k \in A_{i}^{1}}\left(h^{k j}(x)-\underline{g}_{i k}(T, x)\right) \leq h^{i j}(x) \leq \min _{l \in A_{j}^{2}}\left(h^{i l}(x)+\bar{g}_{j l}(T, x)\right) .
$$

To begin with let us point out that the non-local terms $\mathcal{I}(t, x, \phi)$ and $\mathcal{I}_{i j}(t, x, \phi)$ introduced previously are well defined under Assumptions (A0) since for any function $\phi$ of class $\mathcal{C}^{1,2}$, by the mean value theorem, we have

$$
\left|\phi(t, x+\beta(x, e))-\phi(t, x)-D_{x} \phi(t, x) \beta(e, x)\right| \leq C_{t, x}^{(1)}|\beta(x, e)|^{2} \leq C_{t, x}^{(1)}(1 \wedge|e|)^{2},
$$

and

$$
\left|\gamma^{i j}(x, e)(\phi(t, x+\beta(x, e))-\phi(t, x))\right| \leq C_{t, x}^{(2)}\left|\beta(x, e) \gamma^{i j}(x, e)\right| \leq C_{t, x}^{(2)}\left(1 \wedge|e|^{2}\right)
$$


where $C_{t, x}^{(1)}$ and $C_{t, x}^{(2)}$ are bounded constants. They are the bounds of the first and second derivatives of $y \mapsto \phi(t, y)$ in $B\left(x, K_{\beta}\right)$ where $K_{\beta}$ is a bound of the function $\beta$.

Let us consider now the following SDE of jump-diffusion type $\left((t, x) \in[0, T] \times \mathbf{R}^{k}\right)$ :

$$
X_{s}^{t, x}=x+\int_{t}^{s} b\left(r, X_{r}^{t, x}\right) d r+\int_{t}^{s} \sigma\left(r, X_{r}^{t, x}\right) d W_{r}+\int_{t}^{s} \int_{E} \beta\left(X_{r-}^{t, x}, e\right) \hat{N}(d r, d e), s \in[t, T]
$$

The existence and uniqueness of the solution $X^{t, x}:=\left(X_{s}^{t, x}\right)_{s \in[t, T]}$ follows from [15].

We now precise the definition of the viscosity solution of system (2.1). First, for a locally bounded function $u:(t, x) \in[0, T] \times \mathbf{R}^{k} \mapsto u(t, x) \in \mathbf{R}$, we define its lower semi-continuous (lsc for short) envelope $u_{*}$, and upper semi-continuous (usc for short) envelope $u^{*}$ as following:

$$
u_{*}(t, x)=\varliminf_{\left(t^{\prime}, x^{\prime}\right) \rightarrow(t, x), t^{\prime}<T} u\left(t^{\prime}, x^{\prime}\right), \quad u^{*}(t, x)=\varlimsup_{\left(t^{\prime}, x^{\prime}\right) \rightarrow(t, x), t^{\prime}<T} u\left(t^{\prime}, x^{\prime}\right) .
$$

Definition 2.1. A function $\vec{u}(t, x)=\left(u^{i j}(t, x)\right)_{(i, j) \in A^{1} \times A^{2}}:[0, T] \times \mathbf{R}^{k} \rightarrow \mathbf{R}^{m_{1} \times m_{2}}$ such that $u^{i j}$ is lsc (resp. usc) and belongs to $\Pi_{g}$, is said to be a viscosity supersolution (resp. subsolution) of (2.1) if for any $(i, j) \in A^{1} \times A^{2}$ :

- $u^{i j}\left(T, x_{0}\right) \geq($ resp. $\leq) h^{i j}\left(x_{0}\right), \forall x_{0} \in \mathbf{R}^{k}$.

- for any $\left(t_{0}, x_{0}\right) \in(0, T) \times \mathbf{R}^{k}$ and any test function $\phi \in \mathcal{C}^{1,2}\left([0, T] \times \mathbf{R}^{k}\right)$ such that $\left(t_{0}, x_{0}\right)$ is a global minimum (resp. maximum) point of $u^{i j}-\phi$ and $u^{i j}\left(t_{0}, x_{0}\right)=\phi\left(t_{0}, x_{0}\right)$,

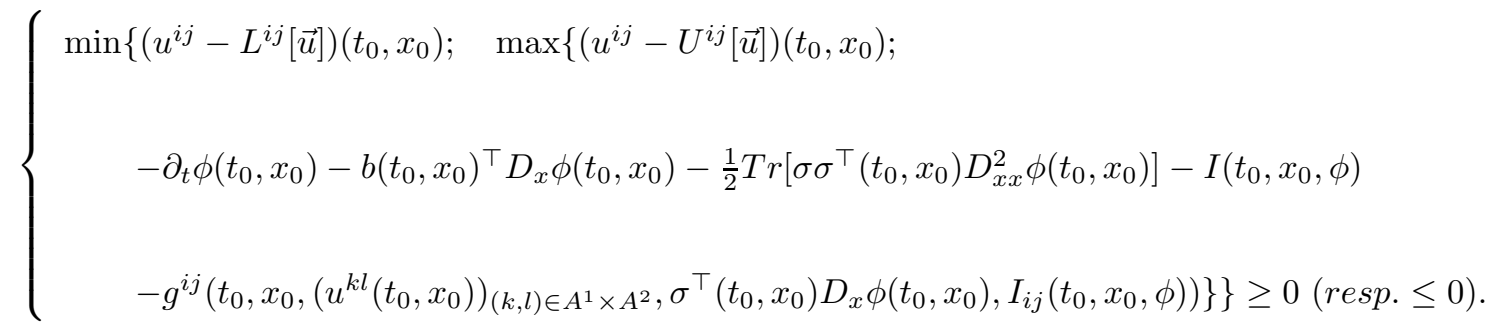

A function $\vec{u}=\left(u^{i j}(t, x)\right)_{(i, j) \in A^{1} \times A^{2}}$ of $\Pi_{g}$ is called a viscosity solution of (2.1) if $\left(u_{*}^{i j}(t, x)\right)_{(i, j) \in A^{1} \times A^{2}}$ (resp. $\left.\left(u_{i j}^{*}(t, x)\right)_{(i, j) \in A^{1} \times A^{2}}\right)$ is a viscosity supersolution (resp. subsolution) of (2.1).

Remark 2.1. By taking $\bar{g}_{j l} \equiv+\infty$ (resp. $\left.\underline{g}_{i k} \equiv+\infty\right)$ for any $j, l \in A^{2}$ (resp. $i, k \in A^{1}$ ) we obtain the definition of a viscosity solution of the system of variational inequalities with interconnected lower (resp. upper) obstacles.

\section{Uniqueness of the viscosity solution of the non-local SVI (2.1)}

In this section we will show the uniqueness of the viscosity solution of (2.1) as a corollary of a comparison result. In the same way with [9], Lemma 4.1, we can prove the following lemma. 
Lemma 3.1. Let $\left(u^{i j}\right)_{(i, j) \in A^{1} \times A^{2}}$ (resp. $\left(w^{i j}\right)_{(i, j) \in A^{1} \times A^{2}}$ ) be an usc subsolution (resp. lsc supersolution) of (2.1) which belongs to $\Pi_{g}$. For $(t, x) \in[0, T] \times R^{k}$, let $\Gamma(t, x)$ be the following set:

$$
\Gamma(t, x):=\left\{(i, j) \in A^{1} \times A^{2}, u^{i j}(t, x)-w^{i j}(t, x)=\max _{(k, l) \in A^{1} \times A^{2}}\left(u^{k l}(t, x)-w^{k l}(t, x)\right)\right\} .
$$

Then there exists $\left(i_{0}, j_{0}\right) \in \Gamma(t, x)$ such that

$$
u^{i_{0} j_{0}}(t, x)>L^{i_{0} j_{0}}[\vec{u}](t, x) \text { and } w^{i_{0} j_{0}}(t, x)<U^{i_{0} j_{0}}[\vec{w}](t, x) .
$$

We now give the comparison result.

Theorem 3.1. Let $\vec{u}=\left(u^{i j}\right)_{(i, j) \in A^{1} \times A^{2}}$ (resp. $\vec{w}=\left(w^{i j}\right)_{(i, j) \in A^{1} \times A^{2}}$ ) be an usc subsolution (resp. lsc supersolution) of (2.1) which belongs to $\Pi_{g}$. Then it holds that:

$$
\forall(i, j) \in \Gamma \text { and }(t, x) \in[0, T] \times \mathbf{R}^{k}, u^{i j}(t, x) \leq w^{i j}(t, x) .
$$

Proof. Let $C$ and $\rho$ be positive constants, which exist thanks to the polynomial growth of $\vec{u}$ and $\vec{w}$, such that for any $(i, j) \in \Gamma$,

$$
\left|u^{i j}(t, x)\right|+\left|w^{i j}(t, x)\right| \leq C\left(1+|x|^{\rho}\right) .
$$

There exists a positive constant $\lambda_{0}$ such that for any $\lambda \geq \lambda_{0}$ and $\theta>0, \tilde{u}^{\theta}:=\left(u^{i j}(t, x)-\theta e^{\lambda t}\left(1+|x|^{2 \rho+2}\right)_{(i, j) \in \Gamma}\right.$ (resp. $\tilde{w}^{\theta}=\left(w^{i j}(t, x)-\theta e^{\lambda t}\left(1+|x|^{2 \rho+2}\right)_{(i, j) \in \Gamma)}\right.$ ) is a subsolution (resp. supersolution) of (2.1) (see [20], pp.1634). Therefore it is enough to show that $\tilde{u}^{\theta} \leq \tilde{w}^{\theta}$ and to take the limit as $\theta \rightarrow 0$ to obtain the desired result. Finally with the previous statement w.l.o.g one can assume that there exists a real constant $\bar{R}>0$ such that for any $|x| \geq \bar{R}, w^{i j}(t, x)>0\left(\operatorname{resp} . u^{i j}(t, x)<0\right)$ for any $(i, j)$ and $t \in[0, T]$.

The proof now will be divided into two steps.

Step 1: Let $C_{i j}$ be the Lipschitz constant of $g^{i j}$ w.r.t. $\vec{y}$. We first assume that there exists a constant $\lambda_{1}>m_{1} m_{2} \cdot \sum_{(i, j) \in \Gamma} C_{i j}$ such that for any $(i, j) \in \Gamma$,

$$
g^{i j}\left(t, x,\left[\vec{y}^{i j}, \zeta_{1}\right], z, q\right)-g^{i j}\left(t, x,\left[\vec{y}^{i j}, \zeta_{2}\right], z, q\right) \leq-\lambda\left(\zeta_{1}-\zeta_{2}\right)
$$

for any $\zeta_{1} \geq \zeta_{2}$ in $\mathbf{R}$ and $(t, x, \vec{y}, z, q)$ in their respective spaces.

We proceed by contradiction. Assume there exists $(\bar{t}, \bar{x}) \in[0, T] \times \mathbf{R}^{k}$ such that:

$$
\max _{(i, j) \in \Gamma}\left(u^{i j}-w^{i j}\right)(\bar{t}, \bar{x})>0 .
$$

Therefore there exists $\left(t^{*}, x^{*}\right) \in\left[0, T\left[\times B(0, \bar{R})\left(B(0, \bar{R})\right.\right.\right.$ is the open ball in $\mathbf{R}^{k}$ centered in 0 and of radius $\bar{R}$ and w.l.o.g. we assume $\left.t^{*}>0\right)$ such that:

$\max _{(t, x) \in[0, T] \times \mathbf{R}^{k}} \max _{(i, j) \in \Gamma}\left\{\left(u^{i j}-w^{i j}\right)(t, x)\right\}=\max _{(t, x) \in[0, T] \times B(0, \bar{R})} \max _{(i, j) \in \Gamma}\left\{\left(u^{i j}-w^{i j}\right)(t, x)\right\}=\max _{(i, j) \in \Gamma}\left\{\left(u^{i j}-w^{i j}\right)\left(t^{*}, x^{*}\right)\right\}>0$. 
Next let $\left(i_{0}, j_{0}\right) \in \Gamma\left(t^{*}, x^{*}\right)$ that satisfies (3.1). For $\epsilon>0, \rho>0$, let $\Phi_{\epsilon, \rho}^{i_{0}, j_{0}}$ be the function defined as follows:

$$
\Phi_{\epsilon, \rho}^{i_{0} j_{0}}(t, x, y):=\left(u^{i_{0} j_{0}}(t, x)-w^{i_{0} j_{0}}(t, y)\right)-\frac{|x-y|^{2}}{\epsilon}-\left|t-t^{*}\right|^{2}-\rho\left|x-x^{*}\right|^{4} .
$$

Let $\left(t_{0}, x_{0}, y_{0}\right)$ be such that

$$
\Phi_{\epsilon, \rho}^{i_{0} j_{0}}\left(t_{0}, x_{0}, y_{0}\right)=\max _{(t, x, y) \in[0, T] \times \bar{B}(0, \bar{R})^{2}} \Phi_{\epsilon, \rho}^{i_{0} j_{0}}(t, x, y)=\max _{(t, x, y) \in[0, T] \times \mathbf{R}^{k+k}} \Phi_{\epsilon, \rho}^{i_{0} j_{0}}(t, x, y) .
$$

The second equality is valid since when $|x| \geq \bar{R}$ (resp. $|y| \geq \bar{R}$ ), $u^{i_{0} j_{0}}(t, x)<0$ (resp. $w^{i_{0} j_{0}}(t, y)>0$ ). On the other hand $\left(t_{0}, x_{0}, y_{0}\right)$ depends actually on $\epsilon$ and $\rho$ which we omit for sake of simplicity. Next as usual we have

$$
\lim _{\epsilon \rightarrow 0}\left(t_{0}, x_{0}, y_{0}\right)=\left(t^{*}, x^{*}, x^{*}\right), \quad \lim _{\epsilon \rightarrow 0} \frac{\left|x_{0}-y_{0}\right|^{2}}{\epsilon}=0,
$$

and

$$
\lim _{\epsilon \rightarrow 0}\left(u^{i_{0} j_{0}}\left(t_{0}, x_{0}\right), w^{i_{0} j_{0}}\left(t_{0}, y_{0}\right)\right)=\left(u^{i_{0} j_{0}}\left(t^{*}, x^{*}\right), w^{i_{0} j_{0}}\left(t^{*}, x^{*}\right)\right) .
$$

Therefore for $\epsilon$ small enough it holds

$$
u^{i_{0} j_{0}}\left(t_{0}, x_{0}\right)>\max _{k \in A_{i_{0}}^{1}}\left(u^{k j_{0}}\left(t_{0}, x_{0}\right)-\underline{g}_{i_{0} k}\left(t_{0}, x_{0}\right)\right)
$$

and

$$
w^{i_{0} j_{0}}\left(t_{0}, y_{0}\right)<\min _{l \in A_{j_{0}}^{2}}\left(w^{i_{0} l}\left(t_{0}, y_{0}\right)+\bar{g}_{j_{0} l}\left(t_{0}, y_{0}\right)\right) .
$$

Once more for $\epsilon$ small enough, we are able to apply Jensen-Ishii's Lemma for non-local operators established by Barles and Imbert ([3], pp. 583) (one can see also [5], Lemma 4.1, pp. 64) with $u^{i_{0} j_{0}}, w^{i_{0} j_{0}}$ and $\phi(t, x, y):=\frac{|x-y|^{2}}{\epsilon}+\left|t-t^{*}\right|^{2}+\rho\left|x-x^{*}\right|^{4}$ at $\left(t_{0}, x_{0}, y_{0}\right)$. For any $\delta \in(0,1)$ there exist $p_{u}^{\epsilon}, q_{u}^{\epsilon}$ in $\mathbf{R}, p_{w}^{\epsilon}, q_{w}^{\epsilon}$ in $\mathbf{R}^{k}$ and $M_{u}^{\epsilon}, M_{w}^{\epsilon}$ two symmetric non-negative matrices of $\mathbf{R}^{k \times k}$ such that:

$$
p_{u}^{\epsilon}-p_{w}^{\epsilon}=\partial_{t} \phi\left(t_{0}, x_{0}, y_{0}\right), \quad q_{u}^{\epsilon}=\partial_{x} \phi\left(t_{0}, x_{0}, y_{0}\right), \quad q_{w}^{\epsilon}=-\partial_{y} \phi\left(t_{0}, x_{0}, y_{0}\right)
$$

and

$$
\left(\begin{array}{cc}
M_{u}^{0} & 0 \\
0 & -M_{w}^{0}
\end{array}\right) \leq\left(\begin{array}{cc}
D_{x x}^{2} \psi_{\rho}\left(t_{0}, x_{0}\right) & 0 \\
0 & 0
\end{array}\right)+\frac{4}{\epsilon}\left(\begin{array}{cc}
I_{k} & -I_{k} \\
-I_{k} & I_{k}
\end{array}\right)
$$

where $\psi_{\rho}(t, x):=\rho\left|x-x^{*}\right|^{4}+\left|t-t^{*}\right|^{2} ;$

$$
\begin{gathered}
\text { (ii) }-p_{u}^{\epsilon}-b\left(t_{0}, x_{0}\right)^{\top} q_{u}^{\epsilon}-\frac{1}{2} \operatorname{Tr}\left(\sigma\left(t_{0}, x_{0}\right)^{\top} M_{u}^{\epsilon} \sigma\left(t_{0}, x_{0}\right)\right)-g^{i_{0} j_{0}}\left(t_{0}, x_{0},\left(u^{i j}\left(t_{0}, x_{0}\right)\right)_{(i, j) \in A^{1} \times A^{2}, \sigma\left(t_{0}, x_{0}\right)^{\top} q_{u}^{\epsilon},}\right. \\
\left.I_{i_{0} j_{0}}^{1, \delta}\left(t_{0}, x_{0}, \phi\left(t_{0}, ., y_{0}\right)\right)+I_{i_{0} j_{0}}^{2, \delta}\left(t_{0}, x_{0}, u^{i_{0} j_{0}}\right)\right)-I_{\delta}^{1}\left(t_{0}, x_{0}, \phi\left(t_{0}, ., y_{0}\right)\right)-I_{\delta}^{2}\left(t_{0}, x_{0}, q_{u}^{\epsilon}, u^{i_{0} j_{0}}\right) \leq 0 ; \\
\text { (iii) }-p_{w}^{\epsilon}-b\left(t_{0}, y_{0}\right)^{\top} q_{w}^{\epsilon}-\frac{1}{2} \operatorname{Tr}\left(\sigma\left(t_{0}, y_{0}\right)^{\top} M_{w}^{\epsilon} \sigma\left(t_{0}, y_{0}\right)\right)-g^{i_{0} j_{0}}\left(t_{0}, y_{0},\left(w^{i j}\left(t_{0}, y_{0}\right)\right)_{(i, j) \in A^{1} \times A^{2}, \sigma\left(t_{0}, y_{0}\right)^{\top} q_{w}^{\epsilon},},\right. \\
\left.\quad I_{i_{0} j_{0}}^{1, \delta}\left(t_{0}, y_{0},-\phi\left(t_{0}, x_{0}, .\right)\right)+I_{i_{0} j_{0}}^{2, \delta}\left(t_{0}, x_{0}, w^{i_{0} j_{0}}\right)\right)-I_{\delta}^{1}\left(t_{0}, y_{0},-\phi\left(t_{0}, x_{0}, .\right)\right)-I_{\delta}^{2}\left(t_{0}, y_{0}, q_{w}^{\epsilon}, w^{i_{0} j_{0}}\right) \geq 0 .
\end{gathered}
$$


Next we are going to provide estimates for the non-local terms. By the same argument as in [20] pp.1645, we have:

$$
\begin{aligned}
I_{\delta}^{2}\left(t_{0}, x_{0}, q_{u}^{\epsilon}, u^{i_{0} j_{0}}\right)-I_{\delta}^{2}\left(t_{0}, y_{0}, q_{w}^{\epsilon}, w^{i_{0} j_{0}}\right) & \leq C \frac{\left|x_{0}-y_{0}\right|^{2}}{\epsilon} \int_{|e| \geq \delta}(1 \wedge|e|)^{2} n(d e)+I_{\delta}^{2}\left(t_{0}, x_{0}, D_{x} \psi_{\rho}\left(t_{0}, x_{0}\right), \psi_{\rho}\right) \\
& \leq C \frac{\left|x_{0}-y_{0}\right|^{2}}{\epsilon}+I_{\delta}^{2}\left(t_{0}, x_{0}, D_{x} \psi_{\rho}\left(t_{0}, x_{0}\right), \psi_{\rho}\right) .
\end{aligned}
$$

On the other hand

$$
D_{x x}^{2} \phi(t, x, y)=2 \epsilon^{-1} I_{k}+D_{x x}^{2} \psi_{\rho}(t, x, y), D_{y y}^{2} \phi(t, x, y)=2 \epsilon^{-1} I_{k}
$$

and by Taylor's expansion we have

$$
\phi(t, x+\beta(x, e), y)-\phi(t, x)-D_{x} \phi(t, x, y) \beta(x, e)=\int_{0}^{1}(1-t) \beta(x, e)^{\top} D_{x x}^{2} \phi(t, x+t \beta(x, e), y) \beta(x, e) d t .
$$

It implies that

$$
I_{\delta}^{1}\left(t_{0}, x_{0}, \phi\left(t_{0}, ., y_{0}\right)\right)=\int_{|e| \leq \delta} n(d e) \int_{0}^{1}(1-t) \beta\left(x_{0}, e\right)^{\top} D_{x x}^{2} \phi\left(t_{0}, x_{0}+t \beta\left(x_{0}, e\right), y_{0}\right) \beta\left(x_{0}, e\right) d t
$$

and similarly

$$
I_{\delta}^{1}\left(t_{0}, y_{0},-\phi\left(t_{0}, x_{0}, .\right)\right)=-\int_{|e| \leq \delta} n(d e) \int_{0}^{1}(1-t) \beta\left(y_{0}, e\right)^{\top} D_{y y}^{2} \phi\left(t_{0}, x_{0}, y_{0}+t \beta\left(y_{0}, e\right)\right) \beta\left(y_{0}, e\right) d t .
$$

Consequently it holds that

$$
\lim _{\delta \rightarrow 0} I_{\delta}^{1}\left(t_{0}, x_{0}, \phi\left(t_{0}, ., y_{0}\right)\right)=\lim _{\delta \rightarrow 0} I_{\delta}^{1}\left(t_{0}, y_{0},-\phi\left(t_{0}, x_{0}, .\right)\right)=0 .
$$

Next by the definition of $\left(t_{0}, x_{0}, y_{0}\right)$, we have

$$
\begin{aligned}
& u^{i_{0} j_{0}}\left(t_{0}, x_{0}+\beta\left(x_{0}, e\right)\right)-u^{i_{0} j_{0}}\left(t_{0}, x_{0}\right) \\
& \leq w^{i_{0}, j_{0}}\left(t_{0}, y_{0}+\beta\left(y_{0}, e\right)\right)-w^{i_{0} j_{0}}\left(t_{0}, y_{0}\right)+\psi_{\rho}\left(t_{0}, x_{0}+\beta\left(x_{0}, e\right)\right)-\psi_{\rho}\left(t_{0}, x_{0}\right) \\
& \quad+\epsilon^{-1}\left\{\left|\beta\left(x_{0}, e\right)-\beta\left(y_{0}, e\right)\right|^{2}-2\left(x_{0}-y_{0}\right)\left(\beta\left(x_{0}, e\right)-\beta\left(y_{0}, e\right)\right)\right\} .
\end{aligned}
$$

Since $\gamma^{i_{0} j_{0}}$ is nonnegative, and by the assumptions on $\beta$ (see (A0)-(i)), for any $\delta>0$,

$$
I_{i_{0} j_{0}}^{2, \delta}\left(t_{0}, x_{0}, u^{i_{0} j_{0}}\right)-I_{i_{0} j_{0}}^{2, \delta}\left(t_{0}, y_{0}, w^{i_{0} j_{0}}\right) \leq I_{i_{0} j_{0}}^{2, \delta}\left(t_{0}, x_{0}, \psi_{\rho}\right)+O\left(\epsilon^{-1}\left|x_{0}-y_{0}\right|^{2}\right),
$$

and it is easy to check that

$$
\left|I_{i_{0}, j_{0}}^{2, \delta}\left(t_{0}, x_{0}, \psi_{\rho}\right)\right| \leq C \rho \int_{|z| \geq \delta} 1 \wedge|e|^{2} n(d e) .
$$

On the other hand, since $\phi$ is a $\mathcal{C}^{2}$-function then, using once more Taylor's expansion to obtain:

$$
\begin{aligned}
& I_{i_{0} j_{0}}^{1, \delta}\left(t_{0}, x_{0}, \phi\left(t_{0}, ., y_{0}\right)\right)=\int_{|e| \leq \delta}\left\{\phi\left(t_{0}, x_{0}+\beta\left(x_{0}, e\right), y_{0}\right)-\phi\left(t_{0}, x_{0}, y_{0}\right)\right\} \gamma^{i_{0} j_{0}}\left(x_{0}, e\right) n(d e) \\
& =\int_{|e| \leq \delta} n(d e) \int_{0}^{1} d t(1-t) \beta\left(x_{0}, e\right)^{\top}\left[\frac{2}{\epsilon}\left\{x_{0}+t \beta\left(x_{0}, e\right)-y_{0}\right\}+D_{x} \psi_{\rho}\left(t_{0}, x_{0}+t \beta\left(x_{0}, e\right)\right)\right] \gamma^{i_{0} j_{0}}\left(x_{0}, e\right) \\
& =\frac{x_{0}-y_{0}}{\epsilon} \int_{|e| \leq \delta} \beta\left(x_{0}, e\right)^{\top} \gamma^{i_{0} j_{0}}\left(x_{0}, e\right) n(d e)+\frac{1}{3} \int_{|e| \leq \delta}\left|\beta\left(x_{0}, e\right)\right|^{2} \gamma^{i_{0} j_{0}}\left(x_{0}, e\right) n(d e) \\
& \quad+\int_{|e| \leq \delta} n(d e) \beta\left(x_{0}, e\right)^{\top} D_{x} \psi_{\rho}\left(t_{0}, x_{0}+t \beta\left(x_{0}, e\right)\right) \gamma^{i_{0} j_{0}}\left(x_{0}, e\right)
\end{aligned}
$$


and

$$
\begin{aligned}
I_{i_{0} j_{0}}^{1, \delta}\left(t_{0}, y_{0},-\phi\left(t_{0}, x_{0}, .\right)\right) & =-\int_{|e| \leq \delta}\left\{\phi\left(t_{0}, x_{0}, y_{0}+\beta\left(y_{0}, e\right)\right)-\phi\left(t_{0}, x_{0}, y_{0}\right)\right\} \gamma^{i_{0} j_{0}}\left(y_{0}, e\right) n(d e) \\
& =\frac{x_{0}-y_{0}}{\epsilon} \int_{|e| \leq \delta} \beta\left(y_{0}, e\right)^{\top} \gamma^{i_{0} j_{0}}\left(y_{0}, e\right) n(d e)-\frac{1}{3} \int_{|e| \leq \delta}\left|\beta\left(x_{0}, e\right)\right|^{2} \gamma^{i_{0} j_{0}}\left(x_{0}, e\right) n(d e)
\end{aligned}
$$

which implies that

$$
\lim _{\delta \rightarrow 0} I_{i_{0} j_{0}}^{1, \delta}\left(t_{0}, x_{0}, \phi\left(t_{0}, ., y_{0}\right)\right)=\lim _{\delta \rightarrow 0} I_{i_{0} j_{0}}^{1, \delta}\left(t_{0}, y_{0},-\phi\left(t_{0}, x_{0}, .\right)\right)=0
$$

Making now the difference between (3.11) and (3.12) yields

$$
\begin{aligned}
& -\left(p_{u}^{\epsilon}-p_{w}^{\epsilon}\right)-\left[b\left(t_{0}, x_{0}\right)^{\top} q_{u}^{\epsilon}-b\left(t_{0}, y_{0}\right)^{\top} q_{w}^{\epsilon}\right]-\frac{1}{2}\left\{\operatorname{Tr}\left[\sigma\left(t_{0}, x_{0}\right)^{\top} M_{u}^{\epsilon} \sigma\left(t_{0}, x_{0}\right)-\sigma\left(t_{0}, y_{0}\right)^{\top} M_{w}^{\epsilon} \sigma\left(t_{0}, y_{0}\right)\right]\right\} \\
& -\left[g^{i_{0} j_{0}}\left(t_{0}, x_{0},\left(u^{i j}\left(t_{0}, x_{0}\right)\right)_{(i, j) \in A^{1} \times A^{2}}, \sigma\left(t_{0}, x_{0}\right)^{\top} q_{u}^{\epsilon}, I_{i_{0} j_{0}}^{1, \delta}\left(t_{0}, x_{0}, \phi\left(t_{0}, ., y_{0}\right)\right)+I_{i_{0} j_{0}}^{2, \delta}\left(t_{0}, x_{0}, u^{i_{0} j_{0}}\right)\right)\right. \\
& \left.-g^{i_{0} j_{0}}\left(t_{0}, y_{0},\left(w^{i j}\left(t_{0}, y_{0}\right)\right)_{(i, j) \in A^{1} \times A^{2}}, \sigma\left(t_{0}, y_{0}\right)^{\top} q_{w}^{\epsilon}, I_{i_{0} j_{0}}^{1, \delta}\left(t_{0}, y_{0},-\phi\left(t_{0}, x_{0}, .\right)\right)+I_{i_{0} j_{0}}^{2, \delta}\left(t_{0}, y_{0}, w^{i_{0} j_{0}}\right)\right)\right] \\
& -I^{1, \delta}\left(t_{0}, x_{0}, \phi\left(t_{0}, ., y_{0}\right)\right)+I^{1, \delta}\left(t_{0}, y_{0},-\phi\left(t_{0}, x_{0}, .\right)\right)-I^{2, \delta}\left(t_{0}, x_{0}, q_{u}^{\epsilon}, u^{i_{0} j_{0}}\right)+I^{2, \delta}\left(t_{0}, y_{0}, q_{w}^{\epsilon}, w^{i_{0} j_{0}}\right) \leq 0 .
\end{aligned}
$$

As $q \in \mathbf{R} \mapsto g^{i j}(t, x, \vec{y}, z, q)$ is non-decreasing and Lipschitz then, by linearization procedure of Lipschitz functions, there exists a bounded non-negative quantity $\Xi$ (which depends on $\epsilon, \delta$, etc.) such that

$$
\begin{aligned}
- & {\left[g^{i_{0} j_{0}}\left(t_{0}, x_{0},\left(u^{i j}\left(t_{0}, x_{0}\right)\right)_{(i, j) \in A^{1} \times A^{2}}, \sigma\left(t_{0}, x_{0}\right)^{\top} q_{u}^{\epsilon}, I_{0_{0} j_{0}}^{1, \delta}\left(t_{0}, x_{0}, \phi\left(t_{0}, ., y_{0}\right)\right)+I_{i_{0} j_{0}}^{2, \delta}\left(t_{0}, x_{0}, u^{i_{0} j_{0}}\right)\right)\right.} \\
& \left.\left.-g^{i_{0} j_{0}}\left(t_{0}, y_{0},\left(w^{i j}\left(t_{0}, y_{0}\right)\right)_{(i, j) \in A^{1} \times A^{2}}, \sigma\left(t_{0}, y_{0}\right)^{\top} q_{w}^{\epsilon}\right), I_{i_{0} j_{0}}^{1, \delta}\left(t_{0}, y_{0},-\phi\left(t_{0}, x_{0}, .\right)\right)+I_{i_{0} j_{0}}^{2, \delta}\left(t_{0}, y_{0}, w^{i_{0} j_{0}}\right)\right)\right] \\
=- & {\left[g^{i_{0} j_{0}}\left(t_{0}, x_{0},\left(u^{i j}\left(t_{0}, x_{0}\right)\right)_{(i, j) \in A^{1} \times A^{2}}, \sigma\left(t_{0}, x_{0}\right)^{\top} q_{u}^{\epsilon}, I_{i_{0} j_{0}}^{1, \delta}\left(t_{0}, x_{0}, \phi\left(t_{0}, ., y_{0}\right)\right)+I_{i_{0} j_{0}}^{2, \delta}\left(t_{0}, x_{0}, u^{i_{0} j_{0}}\right)\right)\right.} \\
& \left.-g^{i_{0} j_{0}}\left(t_{0}, y_{0},\left(w^{i j}\left(t_{0}, y_{0}\right)\right)_{(i, j) \in A^{1} \times A^{2}}, \sigma\left(t_{0}, y_{0}\right)^{\top} q_{w}^{\epsilon}, I_{i_{0} j_{0}}^{1, \delta}\left(t_{0}, x_{0}, \phi\left(t_{0}, ., y_{0}\right)\right)+I_{i_{0} j_{0}}^{2, \delta}\left(t_{0}, x_{0}, u^{i_{0} j_{0}}\right)\right)\right] \\
& -\Xi \times\left[I_{i_{0} j_{0}}^{1, \delta}\left(t_{0}, x_{0}, \phi\left(t_{0}, ., y_{0}\right)\right)+I_{i_{0} j_{0}}^{2, \delta}\left(t_{0}, x_{0}, u^{i_{0} j_{0}}\right)-I_{i_{0} j_{0}}^{1, \delta}\left(t_{0}, y_{0},-\phi\left(t_{0}, x_{0}, .\right)\right)-I_{i_{0} j_{0}}^{2, \delta}\left(t_{0}, y_{0}, w^{i_{0} j_{0}}\right)\right]
\end{aligned}
$$

On the other hand, once more by a linearization procedure and taking into account of (3.2), the first term of the right-hand side of the previous equality verifies:

$$
\begin{aligned}
& {\left[g^{i_{0} j_{0}}\left(t_{0}, x_{0},\left(u^{i j}\left(t_{0}, x_{0}\right)\right)_{(i, j) \in A^{1} \times A^{2}}, \sigma\left(t_{0}, x_{0}\right)^{\top} q_{u}^{\epsilon}, I_{i_{0} j_{0}}^{1, \delta}\left(t_{0}, x_{0}, \phi\left(t_{0}, . ., y_{0}\right)\right)+I_{i_{0} j_{0}}^{2, \delta}\left(t_{0}, x_{0}, u^{i_{0} j_{0}}\right)\right)\right.} \\
& \left.-g^{i_{0} j_{0}}\left(t_{0}, y_{0},\left(w^{i j}\left(t_{0}, y_{0}\right)\right)_{(i, j) \in A^{1} \times A^{2}}, \sigma\left(t_{0}, y_{0}\right)^{\top} q_{w}^{\epsilon}, I_{i_{0} j_{0}}^{1, \delta}\left(t_{0}, x_{0}, \phi\left(t_{0}, ., y_{0}\right)\right)+I_{i_{0} j_{0}}^{2, \delta}\left(t_{0}, x_{0}, u^{i_{0} j_{0}}\right)\right)\right] \\
& \leq-\lambda\left(u^{i_{0} j_{0}}\left(t_{0}, x_{0}\right)-w^{i_{0} j_{0}}\left(t_{0}, y_{0}\right)\right)+\sum_{(i, j) \neq\left(i_{0}, j_{0}\right)} \xi_{t_{0}, x_{0}, y_{0}, \delta}^{i j}\left(u^{i j}\left(t_{0}, x_{0}\right)-w^{i j}\left(t_{0}, y_{0}\right)\right) \\
& +\eta_{t_{0}, x_{0}, y_{0}, \delta}^{i_{0} j_{0}}\left(\sigma\left(t_{0}, x_{0}\right)^{\top} q^{\epsilon}-\sigma\left(t_{0}, y_{0}\right)^{\top} q_{w}^{\epsilon}\right)+\sup _{\vec{y}, z, q}\left|g^{i_{0} j_{0}}\left(t_{0}, x_{0}, \vec{y}, z, q\right)-g^{i_{0} j_{0}}\left(t_{0}, y_{0}, \vec{y}, z, q\right)\right|
\end{aligned}
$$

where $\xi_{t_{0}, x_{0}, y_{0}, \delta}^{i j}$ is bounded non-negative quantity (positiveness stems from (A2)) by $C_{i_{0} j_{0}}$, and $\eta_{t_{0}, x_{0}, y_{0}, \delta}^{i j}$ is 
a bounded quantity by the Lipschitz constant of $g^{i_{0} j_{0}}$ w.r.t. $z$. Therefore from (3.20) we deduce:

$$
\begin{aligned}
& \lambda\left(u^{i_{0} j_{0}}\left(t_{0}, x_{0}\right)-w^{i_{0} j_{0}}\left(t_{0}, y_{0}\right)\right) \leq \\
& \quad\left(p_{u}^{\epsilon}-p_{w}^{\epsilon}\right)+\left[b\left(t_{0}, x_{0}\right)^{\top} q_{u}^{\epsilon}-b\left(t_{0}, y_{0}\right)^{\top} q_{w}^{\epsilon}\right]+\frac{1}{2}\left\{\operatorname{Tr}\left[\sigma\left(t_{0}, x_{0}\right)^{\top} M_{u}^{\epsilon} \sigma\left(t_{0}, x_{0}\right)-\sigma\left(t_{0}, y_{0}\right)^{\top} M_{w}^{\epsilon} \sigma\left(t_{0}, y_{0}\right)\right]\right\} \\
& \quad+\Xi \cdot\left[I_{i_{0} j_{0}}^{1, \delta}\left(t_{0}, x_{0}, \phi\left(t_{0}, . ., y_{0}\right)\right)-I_{i_{0} j_{0}}^{1, \delta}\left(t_{0}, y_{0},-\phi\left(t_{0}, x_{0}, .\right)\right)+I_{i_{0} j_{0}}^{2, \delta}\left(t_{0}, x_{0}, u^{i_{0} j_{0}}\right)-I_{i_{0} j_{0}}^{2, \delta}\left(t_{0}, y_{0}, w^{i_{0} j_{0}}\right)\right] \\
& \quad+I^{1, \delta}\left(t_{0}, x_{0}, \phi\left(t_{0}, ., y_{0}\right)\right)-I^{1, \delta}\left(t_{0}, y_{0},-\phi\left(t_{0}, x_{0}, .\right)\right)+I^{2, \delta}\left(t_{0}, x_{0}, q_{u}^{\epsilon}, u^{i_{0} j_{0}}\right)-I^{2, \delta}\left(t_{0}, y_{0}, q_{w}^{\epsilon}, w^{i_{0} j_{0}}\right) \\
& \quad+\sum_{(i, j) \neq\left(i_{0}, j_{0}\right)} \xi_{t_{0}, x_{0}, y_{0}}\left(u^{i j}\left(t_{0}, x_{0}\right)-w^{i j}\left(t_{0}, y_{0}\right)\right)+\eta_{t_{0}, x_{0}, y_{0}}^{i_{0} j_{0}}\left(\sigma\left(t_{0}, x_{0}\right)^{\top} q^{\epsilon}-\sigma\left(t_{0}, y_{0}\right)^{\top} q_{w}^{\epsilon}\right) \\
& \quad+\sup _{\vec{y}, z, q}\left|g^{i_{0} j_{0}}\left(t_{0}, x_{0}, \vec{y}, z, q\right)-g^{i_{0} j_{0}}\left(t_{0}, y_{0}, \vec{y}, z, q\right)\right| .
\end{aligned}
$$

Next recall (3.13), (3.14),(3.15)-(ii) and (3.19), then take the limit superior as $\delta \rightarrow 0$ then the limit superior as $\rho \rightarrow 0$ to obtain:

$$
\begin{aligned}
& \lambda\left(u^{i_{0} j_{0}}\left(t_{0}, x_{0}\right)-w^{i_{0} j_{0}}\left(t_{0}, y_{0}\right)\right) \leq \\
& \quad\left(p_{u}^{\epsilon}-p_{w}^{\epsilon}\right)+\left[b\left(t_{0}, x_{0}\right)^{\top} q_{u}^{\epsilon}-b\left(t_{0}, y_{0}\right)^{\top} q_{w}^{\epsilon}\right]+\frac{1}{2}\left\{\operatorname{Tr}\left[\sigma\left(t_{0}, x_{0}\right)^{\top} M_{u}^{\epsilon} \sigma\left(t_{0}, x_{0}\right)-\sigma\left(t_{0}, y_{0}\right)^{\top} M_{w}^{\epsilon} \sigma\left(t_{0}, y_{0}\right)\right]\right\} \\
& \quad+\sum_{(i, j) \neq\left(i_{0}, j_{0}\right)} C_{i j}\left(u^{i j}\left(t_{0}, x_{0}\right)-w^{i j}\left(t_{0}, y_{0}\right)\right)^{+}+C_{i_{0} j_{0}}\left(\sigma\left(t_{0}, x_{0}\right)^{\top} q^{\epsilon}-\sigma\left(t_{0}, y_{0}\right)^{\top} q_{w}^{\epsilon}\right) \\
& \quad+\sup _{\vec{y}, z, q}\left|g^{i_{0} j_{0}}\left(t_{0}, x_{0}, \vec{y}, z, q\right)-g^{i_{0} j_{0}}\left(t_{0}, y_{0}, \vec{y}, z, q\right)\right|+\left|O\left(\epsilon^{-1}\left|x_{0}-y_{0}\right|^{2}\right)\right|+C \epsilon^{-1}\left|x_{0}-y_{0}\right|^{2} .
\end{aligned}
$$

Finally by the continuity of $g^{i_{0} j_{0}}$ (see (A1)-(i)), using the properties satisfied by $p_{u}^{\epsilon}, p_{w}^{\epsilon}, q_{u}^{\epsilon}, q_{w}^{\epsilon}, M_{u}^{\epsilon}$ and $M_{w}^{\epsilon}$, sending $\epsilon$ to 0 and taking into account of (3.5)-(3.6) to obtain (in a classical way) that:

$$
\lambda\left(u^{i_{0} j_{0}}\left(t^{*}, x^{*}\right)-w^{i_{0} j_{0}}\left(t^{*}, x^{*}\right)\right) \leq \sum_{(i, j) \neq\left(i_{0}, j_{0}\right)} C_{i j}\left(u^{i j}\left(t^{*}, x^{*}\right)-w^{i j}\left(t^{*}, x^{*}\right)\right)^{+}
$$

which is contradictory by the definitions of $\lambda$ and $\left(t^{*}, x^{*}\right)$. Thus for any $(i, j) \in A^{1} \times A^{2}, u^{i j} \leq w^{i j}$. Note that we have used the fact that $u^{i j}$ (resp $w^{i j}$ ) is usc (resp. lsc) when we take the limit as $\epsilon \rightarrow 0$ to deduce the last inequality.

Step 2: The general case. If $\left(u^{i j}\right)_{(i, j) \in A^{1} \times A^{2}}$ (resp. $\left.\left(w^{i j}\right)_{(i, j) \in A^{1} \times A^{2}}\right)$ is a subsolution (resp. supersolution) of (2.1) in the class $\Pi_{g}$, then for any $\lambda \in \mathbf{R},\left(e^{-\lambda t} u^{i j}\right)_{(i, j) \in A^{1} \times A^{2}}\left(\right.$ resp. $\left.\left(e^{-\lambda t} w^{i j}\right)_{(i, j) \in A^{1} \times A^{2}}\right)$ is a subsolution (resp. supersolution) of a system of type (2.1) but associated with

$\left\{\left(e^{-\lambda t} g^{i j}\left(t, x,\left(e^{\lambda t} u^{k l}\right)_{(k, l) \in A^{1} \times A^{2}}, e^{\lambda t} \sigma(t, x)^{\top} D_{x} u^{i j}(t, x), I_{i j}\left(t, x, e^{\lambda t} u^{i j}\right)\right)-\lambda u^{i j}(t, x)\right.\right.$

$\left.\left.\left(e^{-\lambda t} \underline{g}_{i k}(t, x)\right)_{i, k \in A^{1}},\left(e^{-\lambda t} \bar{g}_{j l}(t, x)\right)_{j, l \in A^{2}},\left(e^{-\lambda T} h^{i j}(x)\right)_{(i, j) \in A^{1} \times A^{2}}\right)\right\}$. But in choosing appropriately the constant $\lambda$ we get that the functions $\tilde{g}^{i j}\left(t, x, \vec{y}, z^{i j}, q\right):=e^{-\lambda t} g^{i j}\left(t, x, e^{\lambda t} \vec{y}, e^{\lambda t} z^{i j}, q\right)-\lambda y^{i j}$ verify (3.2). Therefore by the result of Step 1 we have for any $(i, j) \in A^{1} \times A^{2}, e^{-\lambda t} u^{i j} \leq e^{-\lambda t} w^{i j}$, whence the desired result.

As a by-product we have:

Corollary 3.1. The system of variational inequalities with bilateral obstacles (2.1) has at most one viscosity solution in the class $\Pi_{g}$ which is moreover necessarily continuous.

Finally a remark in the case when we have only lower or upper interconnected obstacles. 
Remark 3.1. If we assume that $\bar{g}_{j k} \equiv+\infty$ (resp. $\left.\underline{q}_{i l} \equiv+\infty\right)$ for any $j, k \in A^{2}$ (res. $\left.i, l \in A^{1}\right)$, then system (2.1) turns into of type with one lower (resp. upper) interconnected obstacles. As the non free loop property is satisfied by $\left(g_{i l}\right)_{i, l \in A^{1}}$ (resp. $\left.\left(\bar{g}_{j k}\right)_{j, k \in A^{2}}\right)$ then the system of variational inequalities with lower (resp. upper) interconnected obstacles has at most one viscosity solution in the class $\Pi_{g}$ which is moreover necessarily continuous.

\section{Approximating schemes and BSDEs}

For $n, m \geq 0$, let $\left(Y^{i j, n, m}, Z^{i j, n, m}, U^{i j, n, m}\right)_{(i, j) \in A^{1} \times A^{2}}$ be the solution of the following system of BSDEs:

$$
\left\{\begin{array}{l}
\left(Y^{i j, n, m}, Z^{i j, n, m}, U^{i j, n, m}\right) \in \mathcal{S}^{2} \times \mathcal{H}^{2} \times \mathcal{H}^{2}(\hat{N}) ; \\
d Y_{s}^{i j, n, m}=-f^{i j, n, m}\left(s, X_{s}^{t, x},\left(Y_{s}^{k l, n, m}\right)_{\left.(k, l) \in A^{1} \times A^{2}, Z_{s}^{i j, n, m}, U_{s}^{i j, n, m}\right) d s+}\right. \\
Z_{s}^{i j, n, m} d B_{s}+\int_{E} U_{s}^{i j, n, m}(e) \hat{N}(d s, d e), s \leq T ; \\
Y_{T}^{i j, n, m}=h^{i j}\left(X_{T}^{t, x}\right),
\end{array}\right.
$$

where

$$
\begin{aligned}
& f^{i j, n, m}\left(s, X_{s}^{t, x},\left(y^{i j}\right)_{(i, j) \in A^{1} \times A^{2}}, z, u\right):=g^{i j, n, m}\left(s, X_{s}^{t, x},\left(y^{k l}\right)_{(k, l) \in A^{1} \times A^{2}}, z, \int_{E} u(e) \gamma^{i j}\left(X_{s}^{t, x}, e\right) n(d e)\right) \\
&=g^{i j}\left(s, X_{s}^{t, x},\left(y^{k l}\right)_{\left.(k, l) \in A^{1} \times A^{2}, z, \int_{E} u(e) \gamma^{i j}\left(X_{s}^{t, x}, e\right) n(d e)\right)}\right. \\
& \quad+n\left(y^{i j}-\max _{k \in A_{i}^{1}}\left\{y^{k j}-\underline{g}_{i k}\left(s, X_{s}^{t, x}\right)\right\}\right)^{-}-m\left(y^{i j}-\min _{l \in A_{j}^{2}}\left\{y^{i l}+\bar{g}_{j l}\left(s, X_{s}^{t, x}\right)\right\}\right)^{+} .
\end{aligned}
$$

Let us notice that under Assumption (A1), the solution $\left(Y^{i j, n, m}, Z^{i j, n, m}, U^{i j, n, m}\right)_{(i, j) \in A^{1} \times A^{2}}$ of $(4.1)$ exists and is unique (see e.g. [29] or [3]). Next, let us focus on the properties of the matrix of processes $\left(Y^{i j, n, m}\right)_{(i, j) \in \Gamma \text {. }}$

Proposition 4.1. For any $(i, j) \in A^{1} \times A^{2}$ and $n, m \geq 0$ we have:

(i)

$$
P-a . s ., \quad Y^{i j, n, m} \leq Y^{i j, n+1, m} \text { and } Y^{i j, n, m+1} \leq Y^{i j, n, m} .
$$

(ii) There exists a deterministic continuous function $v^{i j, n, m} \in \Pi_{g}$ s.t., for any $(t, x) \in[0, T] \times \mathbf{R}^{k}$,

$$
Y_{s}^{i j, n, m}=v^{i j, n, m}\left(s, X_{s}^{t, x}\right), \quad s \in[t, T] .
$$

(iii) for any $(t, x) \in[0, T] \times R^{k}$,

$$
v^{i j, n, m}(t, x) \leq v^{i j, n+1, m}(t, x) \text { and } v^{i j, n, m+1}(t, x) \leq v^{i j, n, m}(t, x) .
$$

Proof. (i) Let $n$ and $m$ be fixed. We are going to use a result by X.Zhu ([31], Theorem 3.1) related to comparison of solutions of multi-dimensional BSDEs with jumps. It is enough to show that for any $t \in$ $[0, T],\left(y_{i j}\right)_{(i, j) \in \Gamma},\left(\bar{y}_{i j}\right)_{(i, j) \in \Gamma} \in \mathbf{R}^{m_{1} \times m_{2}},\left(z_{i j}\right)_{(i, j) \in \Gamma},\left(\bar{z}_{i j}\right)_{(i, j) \in \Gamma} \in\left(\mathbf{R}^{d}\right)^{m_{1} \times m_{2}}$ and $\left(u_{i j}\right)_{(i, j) \in \Gamma},\left(\bar{u}_{i j}\right)_{(i, j) \in \Gamma} \in$ 
$\left(\mathcal{L}_{\mathbf{R}}^{2}\left(E, \mathcal{B}_{E}, n\right)^{m_{1} \times m_{2}}\right.$, there exists a constant $C$ such that:

$$
\begin{aligned}
& -4 \sum_{(i, j) \in \Gamma} y_{i j}^{-}\left\{f^{i j, n+1, m}\left(s, X_{s}^{t, x},\left(y_{k l}^{+}+\bar{y}_{k l}\right)_{(k, l) \in \Gamma}, z_{i j}, u_{i j}\right)-f^{i j, n, m}\left(s, X_{s}^{t, x},\left(\bar{y}_{k l}\right)_{(k, l) \in \Gamma}, \bar{z}_{i j}, \bar{u}_{i j}\right)\right\} \\
& \leq 2 \sum_{(i, j) \in \Gamma} \mathbb{1}_{\left\{y^{i j}<0\right\}}\left|z_{i j}-\bar{z}_{i j}\right|^{2}+C \sum_{(i, j) \in A^{1} \times A^{2}}\left(y_{i j}^{-}\right)^{2} \\
& \quad+2 \sum_{(i, j) \in \Gamma} \int_{E} \mathbb{1}_{\left\{y^{i j} \geq 0\right\}}\left|\left(y_{i j}+u_{i j}(e)-\bar{u}_{i j}(e)\right)^{-}\right|^{2} n(d e) \\
& \quad+2 \sum_{(i, j) \in \Gamma} \int_{E} \mathbb{1}_{\left\{y^{i j}<0\right\}}\left[\left|\left(y_{i j}+u_{i j}(e)-\bar{u}_{i j}(e)\right)^{-}\right|^{2}-\left|y_{i j}^{-}\right|^{2}-2 y_{i j}\left(u_{i j}(e)-\bar{u}_{i j}(e)\right)\right] n(d e)
\end{aligned}
$$

where for any $x \in \mathbf{R}, x^{+}=x \vee 0$ and $x^{-}=(-x) \vee 0$. But the above inequality follows from the facts that for any $(i, j) \in \Gamma$ :

a) $f^{i j, n, m} \leq f^{i j, n+1, m}$;

b) For any $\left(\theta_{k l}\right)_{(k, l) \in \Gamma} \in \mathbf{R}^{m_{1} \times m_{2}}$ such that $\theta_{k l} \geq 0$ if $\left.(k, l) \in \Gamma^{-(i, j)}\right)$ and $\theta_{i j}=0$,

$$
f^{i j, n, m}\left(s, X_{s}^{t, x},\left(y_{k l}+\theta_{k l}\right)_{(k, l) \in \Gamma}, z_{i j}, u_{i j}\right) \geq f^{i j, n, m}\left(s, X_{s}^{t, x},\left(y_{k l}\right)_{(k, l) \in \Gamma}, z_{i j}, u_{i j}\right) .
$$

c) $f^{i j}$ depends only on $z_{i j}, u_{i j}$ and not on the other components $z_{k l}, u_{k l},(k, l) \neq(i, j)$.

d)

$$
\begin{aligned}
& -4 y_{i j}^{-}\left(f^{i j, n, m}\left(s, X_{s}^{t, x},\left(\bar{y}_{k l}\right)_{(k, l) \in \Gamma}, \bar{z}_{i j}, u_{i j}\right)-f^{i j, n, m}\left(s, X_{s}^{t, x},\left(\bar{y}_{k l}\right)_{(k, l) \in \Gamma}, \bar{z}_{i j}, \bar{u}_{i j}\right)\right) \\
& \leq C \\
& \quad\left(y_{i j}^{-}\right)^{2}+2 \int_{E} \mathbb{1}_{\left\{y_{i j} \geq 0\right\}}\left|\left(y_{i j}+u_{i j}(e)-\bar{u}_{i j}(e)\right)^{-}\right|^{2} n(d e) \\
& \quad+2 \int_{E} \mathbb{1}_{\left\{y_{i j}<0\right\}}\left[\left|\left(y_{i j}+u_{i j}(e)-\bar{u}_{i j}(e)\right)^{-}\right|^{2}-\left|y_{i j}^{-}\right|^{2}-2 y_{i j}\left(u_{i j}(e)-\bar{u}_{i j}(e)\right)\right] n(d e)
\end{aligned}
$$

Indeed a), b) and c) are easy to check. We just need to prove d). In the case when $y_{i j} \geq 0$, this inequality is obvious. Next let us focus on the case when $y_{i j}<0$. First note that by a linearization procedure we have:

$$
\begin{aligned}
& -4 y_{i j}^{-}\left(f^{i j, n, m}\left(s, X_{s}^{t, x},\left(\bar{y}_{k l}\right)_{(k, l) \in \Gamma}, \bar{z}_{i j}, u_{i j}\right)-f^{i j, n, m}\left(s, X_{s}^{t, x},\left(\bar{y}_{k l}\right)_{(k, l) \in \Gamma}, \bar{z}_{i j}, \bar{u}_{i j}\right)\right) \\
& =-4 y_{i j}^{-}\left(g^{i j}\left(s, X_{s}^{t, x},\left(\bar{y}_{k l}\right)_{(k, l) \in \Gamma}, \bar{z}_{i j}, \int_{E} \gamma^{i j}\left(X_{s}^{t, x}, e\right) u_{i j}(e) n(d e)\right)\right. \\
& \left.-g^{i j}\left(s, X_{s}^{t, x},\left(\bar{y}_{k l}\right)_{(k, l) \in \Gamma}, \bar{z}_{i j}, \int_{E} \gamma^{i j}\left(X_{s}^{t, x}, e\right) \bar{u}_{i j}(e) n(d e)\right)\right) \\
& =4 y_{i j}^{-} \times \Xi_{1} \times \int_{E} \gamma^{i j}\left(X_{s}^{t, x}, e\right)\left(\bar{u}_{i j}(e)-u_{i j}(e)\right) n(d e)
\end{aligned}
$$

where $\Xi_{1}$ is a non-negative quantity (since $g^{i j}$ is nondecreasing in $q$ ) and bounded by the Lipschitz constant of $g^{i j}$ w.r.t. $q$ and which depends on the other variables. Now

$$
\begin{aligned}
& 4 y_{i j}^{-} \times \Xi_{1} \times \int_{E} \gamma^{i j}\left(X_{s}^{t, x}, e\right)\left(\bar{u}_{i j}(e)-u_{i j}(e)\right) n(d e) \\
& =4 y_{i j}^{-} \times \Xi_{1} \times \int_{u_{i j}-\bar{u}_{i j}<-y_{i j}} \gamma^{i j}\left(X_{s}^{t, x}, e\right)\left(\bar{u}_{i j}(e)-u_{i j}(e)\right) n(d e) \\
& +4 y_{i j}^{-} \times \Xi_{1} \times \int_{u_{i j}-\bar{u}_{i j} \geq-y_{i j}} \gamma^{i j}\left(X_{s}^{t, x}, e\right)\left(\bar{u}_{i j}(e)-u_{i j}(e)\right) n(d e) \\
& \leq 4 y_{i j}^{-} \times \Xi_{1} \times \int_{u_{i j}-\bar{u}_{i j}<-y_{i j}} \gamma^{i j}\left(X_{s}^{t, x}, e\right)\left(\bar{u}_{i j}(e)-u_{i j}(e)\right) n(d e) .
\end{aligned}
$$


But for an appropriate constant $C$,

$$
\gamma^{i j}\left(X_{s}^{t, x}, e\right)\left(\bar{u}_{i j}(e)-u_{i j}(e)\right) \leq C\left(\gamma^{i j}\left(X_{s}^{t, x}, e\right)\right)^{2}+2\left(\bar{u}_{i j}(e)-u_{i j}(e)\right)^{2}
$$

then

$$
\begin{aligned}
& 4 y_{i j}^{-} \times \Xi_{1} \times \int_{E} \gamma^{i j}\left(X_{s}^{t, x}, e\right)\left(\bar{u}_{i j}(e)-u_{i j}(e)\right) n(d e) \\
& \leq C\left(y_{i j}^{-}\right)^{2}+2 \int_{u_{i j}-\bar{u}_{i j}<-y_{i j}} \mathbb{1}_{\left\{y^{i j}<0\right\}}\left[\left|\left(y_{i j}+u_{i j}(e)-\bar{u}_{i j}(e)\right)^{-}\right|^{2}-\left|y_{i j}^{-}\right|^{2}-2 y_{i j}\left(u_{i j}(e)-\bar{u}_{i j}(e)\right)\right] n(d e) \\
& \leq C\left(y_{i j}^{-}\right)^{2}+2 \int_{E} \mathbb{1}_{\left\{y^{i j}<0\right\}}\left[\left|\left(y_{i j}+u_{i j}(e)-\bar{u}_{i j}(e)\right)^{-}\right|^{2}-\left|y_{i j}^{-}\right|^{2}-2 y_{i j}\left(u_{i j}(e)-\bar{u}_{i j}(e)\right)\right] n(d e)
\end{aligned}
$$

which is the claim. Thus $P-a . s ., Y^{i j, n, m} \leq Y^{i j, n+1, m}$. In the same way we have also $P-a . s ., Y^{i j, n, m+1} \leq$ $Y^{i j, n, m}$.

The second claim is just the representation of solutions of standard BSDEs with jumps by deterministic functions in the Markovian framework (see [3]). The inequalities of (4.4) are obtained by taking $s=t$ in (4.2) in view of the representation (4.3) of $Y^{i j, n, m}$ by $v^{i j, n, m}$ and $X^{t, x}$.

Remark 4.1. For any $(i, j) \in \Gamma, v^{i j, n, m}$ is the unique viscosity solution in $\Pi_{g}$ of the following integral-partial differential equation:

$$
\left\{\begin{array}{l}
-\partial_{t} v^{i j, n, m}(t, x)-\mathcal{L} v^{i j, n, m}(t, x)-n\left(v^{i j, n, m}(t, x)-\max _{k \in A_{i}^{1}}\left(v^{k j, n, m}(t, x)-\underline{g}_{i k}(t, x)\right)^{-}\right. \\
+m\left(v^{i j, n, m}(t, x)-\min _{l \in A_{j}^{2}}\left(v^{i l, n, m}(t, x)+\bar{g}_{j l}(t, x)\right)^{+}\right. \\
\quad-g^{i j}\left(t, x,\left(v^{k l, n, m}(t, x)\right)_{(k, l) \in \Gamma}, \sigma(t, x)^{\top} D_{x} v^{i j, n, m}(t, x), I_{i j}\left(t, x, v^{i j, n, m}\right)\right)=0 \\
v^{i j, n, m}(T, x)=h^{i j}(x) .
\end{array}\right.
$$

For more details one can see [3].

We now suggest two approximation schemes obtained from the sequence $\left(\left(Y^{i j, n, m}\right)_{(i, j) \in \Gamma}\right)_{n, m}$ of the solution of system (4.1). The first scheme is a sequence of decreasing reflected BSDEs with interconnected lower obstacles defined as: $\forall(i, j) \in A^{1} \times A^{2}$,

$$
\left\{\begin{array}{c}
\left(\bar{Y}^{i j, m}, \bar{Z}^{i j, m}, \bar{U}^{i j, m}, \bar{K}^{i j, m}\right) \in \mathcal{S}^{2} \times \mathcal{H}^{2} \times \mathcal{H}^{2}(\hat{N}) \times \mathcal{A}^{2} ; \\
\bar{Y}_{s}^{i j, m}=h^{i j}\left(X_{T}^{t, x}\right)+\int_{s}^{T} \bar{f}^{i j, m}\left(r, X_{r}^{t, x},\left(\bar{Y}_{r}^{k l, m}\right)_{(k, l) \in \Gamma}, \bar{Z}_{r}^{i j, m}, \bar{U}_{r}^{i j, m}\right) d r-\int_{s}^{T} \bar{Z}_{r}^{i j, m} d B_{r} \\
\quad-\int_{s}^{T} \int_{E} \bar{U}_{r}^{i j, m}(e) \hat{N}(d r, d e)+\bar{K}_{T}^{i j, m}-\bar{K}_{s}^{i j, m}, \quad s \leq T ; \\
\bar{Y}_{s}^{i j, m} \geq \max _{k \in A_{i}^{1}}\left\{\bar{Y}_{s}^{k j, m}-\underline{g}_{i k}\left(s, X_{s}^{t, x}\right)\right\}, \quad s \leq T ; \\
\int_{0}^{T}\left(\bar{Y}_{s}^{i j, m}-\max _{k \in A_{i}^{1}}\left\{\bar{Y}_{s}^{k j, m}-\underline{g}_{i k}\left(s, X_{s}^{t, x}\right)\right\}\right) d \bar{K}_{s}^{i j, m}=0,
\end{array}\right.
$$


where for any $(i, j) \in \Gamma, m \geq 0$ and $(s, \vec{y}, z, u)\left(u \in \mathcal{L}_{\mathbf{R}}^{2}\left(E, \mathcal{B}_{E}, n\right)\right)$,

$$
\begin{aligned}
\bar{f}^{i j, m}\left(s, X_{s}^{t, x}, \vec{y}, z, u\right):=g^{i j,+, m}\left(s, X_{s}^{t, x},\left(y^{k l}\right)_{\left.(k, l) \in A^{1} \times A^{2}, z, \int_{E} u(e) \gamma^{i j}\left(X_{s}^{t, x}, e\right) n(d e)\right)}\right. \\
:=g^{i j}\left(s, X_{s}^{t, x},\left(y^{k l}\right)_{\left.(k, l) \in A^{1} \times A^{2}, z, \int_{E} u(e) \gamma^{i j}\left(X_{s}^{t, x}, e\right) n(d e)\right)}-m\left(y^{i j}-\min _{l \in A_{j}^{2}}\left(y^{i l}+\bar{g}_{j l}\left(s, X_{s}^{t, x}\right)\right)\right)^{+} .\right.
\end{aligned}
$$

The following result is related to existence and uniqueness of the solution of (4.6) and some of its properties.

Proposition 4.2. i) For any fixed $m \geq 0$, the solution $\left(\bar{Y}^{i j, m}, \bar{Z}^{i j, m}, \bar{U}^{i j, m}, \bar{K}^{i j, m}\right)_{(i, j) \in \Gamma}$ of the system (4.6) exists and is unique. Moreover for any $(i, j)$ and $m \geq 0$, we have:

$$
\lim _{n \rightarrow \infty} \mathbb{E}\left[\sup _{t \leq s \leq T}\left|Y_{s}^{i j, n, m}-\bar{Y}_{s}^{i j, m}\right|^{2}\right] \rightarrow 0 \text { and } P-\text { a.s. }, \quad \bar{Y}^{i j, m} \geq \bar{Y}^{i j, m+1} .
$$

(ii) There exists a unique $m_{1} \times m_{2}$-uplet of deterministic continuous functions $\left(\bar{u}^{k l, m}\right)_{(k, l) \in A^{1} \times A^{2}}$ in $\Pi_{g}$ such that, for every $t \leq T$,

$$
\bar{Y}_{s}^{i j, m}=\bar{u}^{i j, m}\left(s, X_{s}^{t, x}\right), s \in[t, T] .
$$

Moreover, for any $(i, j) \in \Gamma$ and $(t, x) \in[0, T] \times \mathbf{R}^{k}, \bar{u}^{i j, m}(t, x) \geq \bar{u}^{i j, m+1}(t, x)$. Finally, $\left(\bar{u}^{i j, m}\right)_{(i, j) \in A^{1} \times A^{2}}$ is a unique viscosity solution in the class $\Pi_{g}$ of the following system of variational inequalities with interconnected obstacles: $\forall(i, j) \in \Gamma$,

$$
\left\{\begin{array}{l}
\min \left\{\bar{u}^{i j, m}(t, x)-\max _{k \in A_{i}^{1}}\left(\bar{u}^{k j, m}(t, x)-\underline{g}_{i k}(t, x)\right) ;-\partial_{t} \bar{u}^{i j, m}(t, x)-\mathcal{L} \bar{u}^{i j, m}(t, x)\right. \\
-g^{i j,+, m}\left(t, x,\left(\bar{u}^{k l, m}(t, x)\right)_{(k, l) \in \Gamma}, \sigma(t, x)^{\top} D_{x} \bar{u}^{i j, m}(t, x), I_{i j}\left(t, x, \bar{u}^{i j, m}\right)\right\}=0 \\
\bar{u}^{i j, m}(T, x)=h^{i j}(x) .
\end{array}\right.
$$

Proof. (i) It is enough to consider the case $m=0$, since for any $(i, j) \in A^{1} \times A^{2}$, the function

$$
\left(s, x,\left(y^{k l}\right)_{(k l) \in A^{1} \times A^{2}}\right) \rightarrow-m\left(y^{i j}-\min _{l \in A_{j}^{2}}\left(y^{i l}+\bar{g}_{j l}(s, x)\right)\right)^{+}
$$

has the same properties as $f^{i j}$ displayed in (A1) and (A2). First and w.l.o.g we may assume that $f^{i j}$ is non-decreasing w.r.t. $y^{k l}$, for any $(k, l) \in \Gamma$, since thanks to assumption (A2), it is enough to multiply the solution by $e^{\lambda t}$, where $\lambda$ is appropriately chosen in order to fall in this latter case, since $f^{i j}$ is Lipschitz w.r.t. the component $y^{i j}$. Now let $H$ (resp. $F$ ) be the following functions:

$$
H(x)=\sum_{(i, j) \in \Gamma}\left|h^{i j}(x)\right| \text { and } F(t, x, y, z, u)=\sum_{(i, j) \in \Gamma}\left|f^{i j}\left(t, x, y I_{m_{1}, m_{2}}, z, u\right)\right|
$$

where $(y, z, u) \in \mathbf{R}^{1+d} \times \mathcal{L}_{\mathbf{R}}^{2}\left(E, \mathcal{B}_{E}, n\right)$ and $I_{m_{1}, m_{2}}$ is the matrix of $m_{1}$ (resp. $\left.m_{2}\right)$ rows (resp. columns) with entries equal to 1 . Let $(\bar{Y}, \bar{Z}, \bar{U})$ be the solution of the following one-dimensional BSDE with jumps associated with $\left(F\left(s, X_{s}^{t, x}, y, z, u\right), H\left(X_{T}^{t, x}\right)\right)$. Next let $n$ be fixed and let us define recursively the sequence $\left(\tilde{Y}^{k, i j, n}\right)_{k \geq 0}$ as follows: for $k=0$ and any $(i, j) \in A_{1} \times A_{2}$, we set $\tilde{Y}^{0, i j, n}:=-\bar{Y}$. For $k \geq 1$, we define 
$\left(\tilde{Y}^{k, i j, n}, Z^{k, i j, n}, U^{k, i j, n}\right) \in \mathcal{S}^{2} \times \mathcal{H}^{2} \times \mathcal{H}^{2}(\hat{N})$ as the solution of the following system of BSDEs: $\forall(i, j) \in$ $A_{1} \times A_{2}$,

$$
\left\{\begin{aligned}
-d \tilde{Y}_{s}^{k, i j, n} & =f^{i j}\left(s, X_{s}^{t, x},\left(\tilde{Y}_{s}^{k-1, p q, n}\right)_{(p, q) \in A^{1} \times A^{2}}, \tilde{Z}_{s}^{k, i j, n}, \tilde{U}_{s}^{k, i j, n}\right) d s \\
& +n\left(\tilde{Y}_{s}^{k, i j, n}-\max _{l \in A_{i}^{1}}\left(\tilde{Y}_{s}^{k-1, l j, n}-\underline{g}_{i l}\left(s, X_{s}^{t, x}\right)\right)\right)^{-} d s-\tilde{Z}_{s}^{k, i j, n} d B_{s}-\int_{E} \tilde{U}_{s}^{k, i j, n}(e) \hat{N}(d s, d e), s \leq T ; \\
\tilde{Y}_{T}^{k, i j, n}= & h^{i j}\left(X_{T}^{t, x}\right) .
\end{aligned}\right.
$$

The solution of (4.11) exists and is unique since it is a decoupled multi-dimensional standard BSDE with a Lipschiz coefficient, noting that $\left(\tilde{Y}_{s}^{k-1, p q, n}\right)_{(p, q) \in A^{1} \times A^{2}}$ is already given. Since $n$ is fixed and the coefficient $\phi^{i j, n}$ defined by:

$\phi^{i j, n}\left(s, \omega,\left(y^{p q}\right)_{(p, q) \in A^{1} \times A^{2}}, z^{i j}, u^{i j}\right):=f^{i j}\left(s, X_{s}^{t, x}(\omega),\left(y^{p q}\right)_{(p, q) \in A^{1} \times A^{2}}, z^{i j}, u^{i j}\right)+n\left(y^{i j}-\max _{l \in A_{i}^{1}}\left(y^{l j}-\underline{g}_{i l}\left(s, X_{s}^{t, x}\right)\right)\right)^{-}$

is Lipschitz w.r.t. $\left(\left(y^{p q}\right)_{(p, q) \in A^{1} \times A^{2}}, z^{i j}, u^{i j}\right)$, the sequence $\left(\tilde{Y}^{k, i j, n}\right)_{k \geq 0}$ converges in $\mathcal{S}^{2}$ to $Y^{i j, n, 0}$ as $k \rightarrow \infty$, for any $i, j$ and $n$. Finally by comparison and an induction argument, used twice (with $n$ and then with $k$ ), we obtain:

$$
\tilde{Y}^{k, i j, n} \leq \tilde{Y}^{k, i j, n+1} \text { and } \tilde{Y}^{k, i j, n} \leq \bar{Y} .
$$

Note that for the second inequality, we take into account of the fact that $n\left(\bar{Y}_{s}-\max _{l \in A_{i}^{1}}\left(\bar{Y}_{s}-\underline{g}_{i l}\left(s, X_{s}^{t, x}\right)\right)\right)^{-} \equiv 0$ since $\underline{g}_{i l} \geq 0$. Take now the limit w.r.t. $k$ in the previous inequalities to obtain:

$$
Y^{i j, n, 0} \leq Y^{i j, n+1,0} \text { and } Y^{i j, n, 0} \leq \bar{Y}
$$

Therefore there exists a progressively measurable process $\bar{Y}^{i j, 0}$ such that

$$
Y^{i j, n, 0} \nearrow \bar{Y}^{i j, 0} \text { and } \bar{Y}^{i j, 0} \leq \bar{Y} \text {. }
$$

Now using the monotonic limit theorem by E.Essaky ([14], Theorem 3.1) there exist $\left(\bar{Z}^{i j, 0}, \bar{U}^{i j, 0}\right) \in \mathcal{H}^{2} \times$ $\mathcal{H}^{2}(\hat{N})$ and $\bar{K}^{i j, 0} \in \mathcal{S}^{2}$ non-decreasing such that:

(a) $\bar{Y}^{i j, 0}$ belongs to $\mathcal{S}^{2}$ and for any stopping time $\tau, \lim _{n \rightarrow \infty} \nearrow Y_{\tau}^{i j, n, 0}=\bar{Y}_{\tau}^{i j, 0}$.

(b) $\bar{K}^{i j, 0}$ is predictable RCLL non-decreasing, $\bar{K}_{0}^{i j, 0}=0$ and for any stopping time $\tau$, the sequence $\left(K_{\tau}^{i j, n, 0}\right)_{n \geq 0}$ converge weakly in $L^{2}(P)$ to $\bar{K}_{\tau}^{i j, 0}$;

(c) For any $p \in[1,2)$,

$$
\lim _{n \rightarrow \infty} \mathbb{E}\left[\int_{0}^{T}\left|Z_{s}^{i j, n, 0}-\bar{Z}^{i j, 0}\right|^{p} d s\right]=0, \quad \lim _{n \rightarrow \infty} \mathbb{E}\left[\int_{0}^{T} \int_{E}\left|U_{s}^{i j, n, 0}-\bar{U}^{i j, 0}\right|^{\frac{p}{2}} n(d e) d s\right]=0 ;
$$

(d) Moreover for any $(i, j) \in \Gamma$ and $s \leq T$ we have:

$$
\left\{\begin{array}{l}
\bar{Y}_{s}^{i j, 0}=h^{i j}\left(X_{T}^{t, x}\right)+\int_{s}^{T} f^{i j}\left(r, X_{r}^{t, x},\left(\bar{Y}_{r}^{k l, 0}\right)_{(k, l) \in \Gamma}, \bar{Z}_{r}^{i j, 0}, \bar{U}_{r}^{i j, 0}\right) d r+\bar{K}_{T}^{i j, 0}-\bar{K}_{s}^{i j, 0} \\
\quad-\int_{s}^{T} \bar{Z}_{r}^{i j, 0} d B_{r}-\int_{s}^{T} \int_{E} \bar{U}_{r}^{i j, 0}(e) n(d e) d r ; \\
\bar{Y}_{s}^{i j, 0} \geq \max _{k \in A_{i}^{1}}\left\{\bar{Y}_{s}^{k j, 0}-\underline{g}_{i k}\left(s, X_{s}^{t, x}\right)\right\} ; \\
\int_{0}^{T}\left(\bar{Y}_{s-}^{i j, 0}-\max _{k \in A_{i}^{1}}\left\{\bar{Y}_{s-}^{k j, 0}-\underline{g}_{i k}\left(s, X_{s-}^{t, x}\right)\right) d \bar{K}_{s}^{i j, 0}=0 .\right.
\end{array}\right.
$$


The remaining of the proof is the same as the one of Theorem 3.2 in [20], pp.1623, i.e., to show that the predictable process $\bar{K}^{i j, 0}$ is continuous thanks to the non free loop property (A4). Thus $\left(\bar{Y}^{i j, 0}, \bar{Z}^{i j, 0}, \bar{U}^{i j, 0}, \bar{K}^{i j, 0}\right)$ is a solution of (4.6) with $m=0$.

Uniqueness of the solution of (4.6) is obtained in the same way as in ([18], pp.193) or ([6], pp.122) in making the connection between the solutions of systems of type (4.6) and the value function of the related optimal switching problem. This is possible since the hypotheses on the data allow for comparison in this framework of Brownian-Poisson noise type (especially (A1)).

Finally the last property of convergence stems from the following facts: (i) $Y^{i j, n, 0} \nearrow_{n} \bar{Y}^{i j, 0}$; (ii) $Y_{-}^{i j, n, 0} \nearrow_{n}$ $\bar{Y}_{-}^{i j, 0}$; (iii) A weak version of Dini's theorem for RCLL process (see [8], pp.202). Note that property (ii) is a consequence of continuity of $\bar{K}^{i j, 0}$ which implies that the predictable projection of $\bar{Y}^{i j, 0}$ is nothing but $\bar{Y}_{-}^{i j, 0}$ and the same holds for $Y^{i j, n, 0}$.

(ii) By (4.3), (4.4) and (4.12), we obtain that the sequence of functions $\left(v^{i j, n, 0}\right)_{n \geq 0}$ is convergent for any $(i, j) \in \Gamma$. So let us set $\bar{u}^{i j, 0}(t, x):=\lim _{n} \nearrow v^{i j, n, 0}(t, x)$. Therefore by (4.3) and (4.8), the relation (4.9) holds true.

Next as previously mentionned, we can obtain the same results for arbitrary $m$ and not only for $m=0$. Therefore we define $\bar{u}^{i j, m}(t, x):=\lim _{n} v^{i j, n, m}(t, x)$. Those functions verify (4.9) and $\bar{u}^{i j, m} \geq \bar{u}^{i j, m+1}$ by (4.4). Next $\left(\bar{u}^{i j, m}\right)_{(i, j) \in A^{1} \times A^{2}}$ is a viscosity solution of (4.10) since $\left(v^{i j, n, m}\right)_{(i, j) \in \Gamma}$ is solution of (4.1) and by arguing as in ([20], Theorem 4.1). Finally uniqueness in the class $\Pi_{g}$ and continuity holds true by Remark 3.1 .

We now consider the increasing approximating scheme: $\forall(i, j) \in \Gamma$,

$$
\left\{\begin{array}{l}
\left(\underline{Y}^{i j, n}, \underline{Z}^{i j, n}, \underline{U}^{i j, n}, \underline{K}^{i j, n}\right) \in \mathcal{S}^{2} \times \mathcal{H}^{2} \times \mathcal{H}^{2}(\hat{N}) \times \mathcal{A}^{2} ; \\
\underline{Y}_{s}^{i j, n}=h^{i j}\left(X_{T}^{t, x}\right)+\int_{s}^{T} \underline{f}^{i j, n}\left(r, X_{r}^{t, x},\left(\underline{Y}_{r}^{k, l, n}\right)_{(k, l) \in \Gamma}, \underline{Z}_{r}^{i j, n}, \underline{U}_{r}^{i j, n}\right) d r-\int_{s}^{T} \underline{Z}_{r}^{i j, n} d B_{r} \\
\quad-\int_{s}^{T} \int_{E} \underline{U}_{r}^{i j, n}(e) \hat{N}(d r, d e)-\left(\underline{K}_{T}^{i j, n}-\underline{K}_{s}^{i j, n}\right), \quad s \leq T ; \\
\underline{Y}_{s}^{i j, n} \leq \min _{l \in A_{j}^{2}}\left\{\underline{Y}_{s}^{i l, n}+\bar{g}_{j l}\left(s, X_{s}^{t, x}\right)\right\}, \quad s \leq T, \\
\int_{0}^{T}\left(\underline{Y}_{s}^{i j, n}-\min _{l \in A_{j}^{2}}\left\{\underline{Y}_{s}^{k j, n}+\bar{g}_{j l}\left(s, X_{s}^{t, x}\right)\right\}\right) d \underline{K}_{s}^{i j, n}=0,
\end{array}\right.
$$

where for any $(i, j) \in \Gamma, n \geq 0$ and $(s, \vec{y}, z, u)$,

$$
\begin{array}{r}
\underline{f}^{i j, n}\left(s, X_{s}^{t, x}, \vec{y}, z, u\right):=g^{i j,-, n}\left(s, X_{s}^{t, x},\left(y^{k l}\right)_{\left.(k, l) \in A^{1} \times A^{2}, z, \int_{E} u(e) \gamma^{i j}\left(X_{s}^{t, x}, e\right) n(d e)\right)}\right. \\
\begin{array}{r}
:=g^{i j}\left(s, X_{s}^{t, x},\left(y^{k l}\right)_{(k, l) \in \Gamma}, z, \int_{E} u(e) \gamma^{i j}\left(X_{s}^{t, x}, e\right) n(d e)\right) \\
+n\left(y^{i j}-\max _{k \in A_{i}^{1}}\left(y^{k j}-\underline{g}_{i k}\left(s, X_{s}^{t, x}\right)\right)\right)^{-} .
\end{array}
\end{array}
$$


The existence of $\left(\underline{Y}^{i j, n}, \underline{Z}^{i j, n}, \underline{U}^{i j, n}, \underline{K}^{i j, n}\right)_{(i, j) \in A^{1} \times A^{2}}$ is obtained thanks to Proposition 4.2 in considering the system of reflected BSDEs with interconnected lower obstacles associated with the data $\left\{\left(-\underline{f}^{i j, n}\left(s, X_{s}^{t, x},-\vec{y},-z,-u\right)\right)_{(i, j) \in A^{1} \times A^{2}},\left(-h^{i j}\right)_{(i, j) \in A^{1} \times A^{2}},\left(\bar{g}_{j l}\right)_{j, l \in A_{2}}\right\}$ which has a unique solution $\left(Y_{1}^{i j, n}, Z_{1}^{i j, n}, U_{1}^{i j, n}, K_{1}^{i j, n}\right)_{(i, j) \in A^{1} \times A^{2}}$ and then it is enough to set $\left(\underline{Y}^{i j, n}, \underline{Z}^{i j, n}, \underline{U}^{i j, n}, \underline{K}^{i j, n}\right)_{(i, j) \in A^{1} \times A^{2}}=$ $\left(-Y_{1}^{i j, n},-Z_{1}^{i j, n},-U_{1}^{i j, n}, K_{1}^{i j, n}\right)_{(i, j) \in A^{1} \times A^{2}}$. The following is the analogous of Proposition 4.2.

Proposition 4.3. i) For any fixed $(i, j) \in \Gamma$ and $n \geq 0$ we have:

$$
\lim _{m \rightarrow \infty} \mathbb{E}\left[\sup _{t \leq s \leq T}\left|Y_{s}^{i j, n, m}-\underline{Y}_{s}^{i j, n}\right|^{2}\right] \rightarrow 0 \text { and } P-a . s ., \quad \underline{Y}^{i j, n} \leq \underline{Y}^{i j, n+1} .
$$

(ii) There exists a unique $m_{1} \times m_{2}$-uplet of deterministic continuous functions $\left(\underline{u}^{k l, n}\right)_{(k, l) \in A^{1} \times A^{2}}$ in $\Pi_{g}$ such that, for every $(t, x) \in[0, T] \times \mathbf{R}^{k}$,

$$
\underline{Y}_{s}^{i j, n}=\underline{u}^{i j, n}\left(s, X_{s}^{t, x}\right), \forall s \in[t, T]
$$

Moreover, for any $(i, j) \in \Gamma$ and $(t, x) \in[0, T] \times \mathbf{R}^{k}, \underline{u}^{i j, n}(t, x) \leq \underline{u}^{i j, n+1}(t, x)$. Finally, $\left(\underline{u}^{i j, n}\right)_{(i, j) \in A^{1} \times A^{2}}$ is the unique viscosity solution in the class $\Pi_{g}$ of the following system of variational inequalities with interconnected upper obstacles. $\forall(i, j) \in \Gamma$,

$$
\left\{\begin{array}{l}
\max \left\{\underline{u}^{i j, n}(t, x)-\min _{l \in A_{j}^{2}}\left(\underline{u}^{i l, n}(t, x)+\bar{g}_{j l}(t, x)\right) ;-\partial_{t} \underline{u}^{i j, n}(t, x)-\mathcal{L} \underline{u}^{i j, n}(t, x)\right. \\
\left.-g^{i j,-, n}\left(t, x,\left(\underline{u}^{k l, n}(t, x)\right)_{(k, l) \in \Gamma}, \sigma(t, x)^{\top} D_{x} \underline{u}^{i j, n}(t, x), I_{i j}\left(t, x, \underline{u}^{i j, n}\right)\right)\right\}=0 \\
\underline{u}^{i j, n}(T, x)=h^{i j}(x) .
\end{array}\right.
$$

Now let us define $\bar{u}^{i j}, \underline{u}^{i j},(i, j) \in \Gamma$, by:

$$
\bar{u}^{i j}(t, x):=\lim _{m \rightarrow \infty} \bar{u}^{i j, m}(t, x), \quad \underline{u}^{i j}(t, x):=\lim _{n \rightarrow \infty} \underline{u}^{i j, n}(t, x),(t, x) \in[0, T] \times \mathbf{R}^{k} .
$$

We then have:

Corollary 4.1. For any $(i, j) \in A^{1} \times A^{2}$, the function $\bar{u}^{i j}$ (resp. $\underline{u}^{i j}$ ) is usc (resp. lsc). Moreover, $\bar{u}^{i j}$ and $\underline{u}^{i j}$ belong to $\Pi_{g}$ and for any $(t, x) \in[0, T] \times \mathbf{R}^{k}$,

$$
\underline{u}^{i j}(t, x) \leq \bar{u}^{i j}(t, x) .
$$

Proof. For any $(i, j) \in A_{1} \times A_{2}$, the function $\bar{u}^{i j}$ (resp. $\underline{u}^{i j}$ ) is obtained as a decreasing (resp. increasing) limit of continuous functions. Therefore, it is usc (resp. $l s c$ ). Next, for any $(i, j)$ and $n, m$,

$$
u^{i j, n, m}(t, x) \leq u^{i j, n, 0}(t, x), \quad(t, x) \in[0, T] \times \mathbf{R}^{k},
$$

as the sequence $\left(u^{i j, n, m}\right)_{m \geq 0}$ is decreasing. Thus, taking the limit as $m \rightarrow \infty$ we obtain,

$$
\underline{u}^{i j, n} \leq u^{i j, n, 0} .
$$

Now using (4.3) and (4.8), it follows that, for any $t \leq T$ and $s \in[t, T], Y_{s}^{i j, n, 0}=u^{i j, n, 0}\left(s, X_{s}^{t, x}\right)$ and the processes $Y^{i j, n, 0}$ converges in $\mathcal{S}^{2}$, as $n \rightarrow \infty$, to $\bar{Y}^{i j, 0}$ which is solution of (4.6) with $m=0$. Furthermore, by 
(4.9), there exists a deterministic continuous function $\bar{u}^{i j, 0}$ with polynomial growth such that for any $t \leq T$ and $s \in[t, T], \bar{Y}_{s}^{i j, 0}=\bar{u}^{i j, 0}\left(s, X_{s}^{t, x}\right)$. Then taking $s=t$ and the limit as $n \rightarrow \infty$ to obtain

$$
\underline{u}^{i j}(t, x):=\lim _{n \rightarrow \infty} \underline{u}^{i j, n}(t, x) \leq \lim _{n \rightarrow \infty} u^{i j, n, 0}(t, x)=\bar{u}^{i j, 0}(t, x), \quad \forall(t, x) \in[0, T] \times \mathbf{R}^{k} .
$$

But $\bar{u}^{i j, 0}$ and $\underline{u}^{i j, n}$ belong to $\Pi_{g}$ and $\underline{u}^{i j, n} \leq \underline{u}^{i j, n+1}$. Thus $\underline{u}^{i j} \in \Pi_{g}$, for any $(i, j) \in A_{1} \times A_{2}$. The last inequality follows from (4.4) and the definitions of $\bar{u}^{i j}$ and $\underline{u}^{i j}$. In the same way one can show that $\bar{u}^{i j} \in \Pi_{g}$, for any $(i, j) \in A_{1} \times A_{2}$.

We now focus on the proof of existence of a solution for system (2.1).

Proposition 4.4. The family $\left(\bar{u}^{i j}\right)_{(i, j) \in A^{1} \times A^{2}}$ is a viscosity subsolution of the system (2.1).

Proof. First recall that for any $(i, j) \in \Gamma, \bar{u}^{i j}=\lim _{m} \searrow \bar{u}^{i j, m}$, so that $\bar{u}^{i j}$ is usc. Moreover since $\bar{u}^{i j, m}(T, x)=$ $h^{i j}(x)$ then $\bar{u}^{i j}(T, x)=h^{i j}(x), \forall x \in \mathbf{R}^{k}$.

Now let $(i, j) \in \Gamma$ and $(t, x) \in(0, T) \times \mathbf{R}^{k}$ be fixed. We suppose that there exists $\epsilon_{0}>0$ s.t.

$$
\bar{u}^{i j}(t, x) \geq L^{i j}[\vec{u}](t, x)+\epsilon_{0}
$$

otherwise the subsolution property holds. Thanks to the decreasing convergence of $\left(\bar{u}^{i j, m}\right)_{m \geq 0}$ to $\bar{u}^{i j}$, there exists $m_{0}$ such that for any $m \geq m_{0}$, we have

$$
\bar{u}^{i j, m}(t, x) \geq L^{i j}\left[\left(\bar{u}^{p q, m}\right)_{(p, q) \in A^{1} \times A^{2}}\right](t, x)+\frac{\epsilon_{0}}{2} .
$$

As for any $m \geq 0, \bar{u}^{i j, m}$ and $L^{i j}\left[\left(\bar{u}^{p q, m}\right)_{(p, q) \in A^{1} \times A^{2}}\right]$ are continuous, then there exists a neighborhood $\Theta_{m}$ of $(t, x)$ such that

$$
\bar{u}^{i j, m}\left(t^{\prime}, x^{\prime}\right) \geq L^{i j}\left[\left(\bar{u}^{p q, m}\right)_{(p, q) \in A^{1} \times A^{2}}\right]\left(t^{\prime}, x^{\prime}\right)+\frac{\epsilon_{0}}{4}, \forall\left(t^{\prime}, x^{\prime}\right) \in \Theta_{m} .
$$

Let now $\phi$ be a $\mathcal{C}^{1,2}$-function such that $\phi(t, x)=\bar{u}^{i j}(t, x)$ and $\bar{u}^{i j}-\phi$ has a global strict maximum in $(t, x)$. Next let $\delta>0$ and for $m \geq 0$ let $\left(t_{m}, x_{m}\right)$ be the global maximum of $\bar{u}^{i j}-\phi$ on $[0, T] \times B^{\prime}\left(x, 2 \delta K_{\beta}\right)$ ( $K_{\beta}$ is a bound for $\beta$ and $B^{\prime}\left(x, 2 \delta K_{\beta}\right)$ is the closure of $B\left(x, 2 \delta K_{\beta}\right)$ ), which exists since the function $\bar{u}^{i j}-\phi$ is usc. But there exists a subsequence $\left\{m_{k}\right\}$ such that

$$
\left(t_{m_{k}}, x_{m_{k}}\right) \rightarrow_{k}(t, x) \text { and } \bar{u}^{i j, m_{k}}\left(t_{m_{k}}, x_{m_{k}}\right) \rightarrow_{k} \bar{u}^{i j}(t, x)
$$

Actually by Lemma 6.1 in [7], there exist a subsequence $\left\{m_{k}\right\}$ and a sequence $\left(t_{m_{k}}^{\prime}, x_{m_{k}}^{\prime}\right)_{k}$ such that

$$
\left(t_{m_{k}}^{\prime}, x_{m_{k}}^{\prime}\right)_{k} \rightarrow_{k}(t, x) \text { and } \bar{u}^{i j, m_{k}}\left(t_{m_{k}}^{\prime}, x_{m_{k}}^{\prime}\right) \rightarrow \bar{u}^{i j}(t, x) .
$$

Next let us consider a convergent subsequent of $\left(t_{m_{k}}, x_{m_{k}}\right)$, which we still denote by $\left(t_{m_{k}}, x_{m_{k}}\right)$, and let $(\bar{t}, \bar{x})$ be its limit. Then for some $k_{0}$ and for $k \geq k_{0}$ we have

$$
\begin{aligned}
& \bar{u}^{i j}(\bar{t}, \bar{x})-\phi(\bar{t}, \bar{x}) \geq \lim \sup _{k}\left(\bar{u}^{i j, m_{k}}\left(t_{m_{k}}, x_{m_{k}}\right)-\phi\left(t_{m_{k}}, x_{m_{k}}\right)\right) \geq \liminf _{k}\left(\bar{u}^{i j, m_{k}}\left(t_{m_{k}}, x_{m_{k}}\right)-\phi\left(t_{m_{k}}, x_{m_{k}}\right)\right) \\
& \quad \geq \liminf _{k}\left(\bar{u}^{i j, m_{k}}\left(t_{m_{k}}^{\prime}, x_{m_{k}}^{\prime}\right)-\phi\left(t_{m_{k}}^{\prime}, x_{m_{k}}^{\prime}\right)\right)=\bar{u}^{i j}(t, x)-\phi(t, x) \geq \bar{u}^{i j}(\bar{t}, \bar{x})-\phi(\bar{t}, \bar{x}) .
\end{aligned}
$$


It implies that $\bar{u}^{i j}(t, x)-\phi(t, x)=\bar{u}^{i j}(\bar{t}, \bar{x})-\phi(\bar{t}, \bar{x})$ then $(t, x)=(\bar{t}, \bar{x})$ since the maximum is strict. On the other hand we obviously have $\bar{u}^{i j, m_{k}}\left(t_{m_{k}}, x_{m_{k}}\right) \rightarrow_{k} \bar{u}^{i j}(t, x)$. Finally since this is valid for any subsequence of $\left(t_{m_{k}}, x_{m_{k}}\right)$, then the claim follows.

But from the subsequence $\left\{m_{k}\right\}$ one can substract a subsequence which we still denote by $\left\{m_{k}\right\}$ such that $\left(t_{m_{k}}, x_{m_{k}}\right)$ belongs to $\Theta_{m_{k}}$. Indeed if this is not possible one can find a subsequence $\left\{m_{p}\right\}$ of $\left\{m_{k}\right\}$ such that for $p \geq 0,\left(t_{m_{p}}, x_{m_{p}}\right)$ does not belong to $\Theta_{m_{p}}$, i.e.,

$$
\bar{u}^{i j, m_{p}}\left(t_{m_{p}}, x_{m_{p}}\right)<L^{i j}\left[\left(\bar{u}^{p q, m_{p}}\right)_{(p, q) \in A^{1} \times A^{2}}\right]\left(t_{m_{p}}, x_{m_{p}}\right)+\frac{\epsilon_{0}}{2} .
$$

Then in taking the limit w.r.t. $p$ we obtain

$$
\bar{u}^{i j}(t, x) \leq\left(L^{i j}\left[\left(\bar{u}^{p q}\right)_{\left.(p, q) \in A^{1} \times A^{2}\right]}\right]\right)^{*}(t, x)+\frac{\epsilon_{0}}{2}
$$

where $(.)^{*}$ stands for the upper semi-continuous envelope. But

$$
\left(L^{i j}\left[\left(\bar{u}^{p q}\right)_{\left.(p, q) \in A^{1} \times A^{2}\right]}\right)^{*}=\max _{k \in A_{1}^{i}}\left(\bar{u}^{k j}-\underline{g}_{i k}\right)^{*}=\max _{k \in A_{1}^{i}}\left(\bar{u}^{k j}-\underline{g}_{i k}\right) .\right.
$$

Therefore we have

$$
\bar{u}^{i j}(t, x) \leq \max _{k \in A_{1}^{i}}\left(\bar{u}^{k j}-\underline{g}_{i k}\right)(t, x)+\frac{\epsilon_{0}}{2}=L^{i j}[\bar{u}](t, x)+\frac{\epsilon_{0}}{2}
$$

which is contradictory with (4.18). Hereafter we consider this subsequence $\left\{m_{k}\right\}$.

Now for $k$ large enough: (i) $\left(t_{m_{k}}, x_{m_{k}}\right) \in(0, T) \times B\left(x, 2 K_{\beta} \delta\right)$ and is the global maximum of $\bar{u}^{i j, m_{k}}-\phi$ in $(0, T) \times B\left(x_{m_{k}}, K_{\beta} \delta\right)$; (ii) $\bar{u}^{i j, m_{k}}\left(t_{m_{k}}, x_{m_{k}}\right)>L^{i j}\left[\left(\bar{u}^{p q, m_{k}}\right)_{(p, q) \in A^{1} \times A^{2}}\right]\left(t_{m_{k}}, x_{m_{k}}\right)$. As $\bar{u}^{i j, m_{k}}$ is a subsolution of (4.10), then by Proposition 5.1 - Remark 5.1 in Appendix, we have

$$
\begin{gathered}
-\partial_{t} \phi\left(t_{m_{k}}, x_{m_{k}}\right)-\overline{\mathcal{L}} \phi\left(t_{m_{k}}, x_{m_{k}}\right)-I_{\delta}^{1}\left(t_{m_{k}}, x_{m_{k}}, \phi\right)-I_{\delta}^{2}\left(t_{m_{k}}, x_{m_{k}}, D_{x} \phi\left(t_{m_{k}}, x_{m_{k}}\right), \bar{u}^{i j, m_{k}}\right) \\
+m_{k}\left(\bar{u}^{i j, m_{k}}\left(t_{m_{k}}, x_{m_{k}}\right)-\min _{l \in A_{2}^{j}}\left(\bar{u}^{i l, m_{k}}\left(t_{m_{k}}, x_{m_{k}}\right)+\bar{g}_{j l}\left(t_{m_{k}}, x_{m_{k}}\right)\right)\right)^{+} \\
\leq g^{i j}\left[t_{m_{k}}, x_{m_{k}},\left(\bar{u}^{p l, m_{k}}\left(t_{m_{k}}, x_{m_{k}}\right)\right)(p, l) \in A^{1} \times A^{2}, \sigma\left(t_{m_{k}}, x_{m_{k}}\right)^{\top} D_{x} \phi\left(t_{m_{k}}, x_{m_{k}}\right),\right. \\
\left.I_{i j}^{1, \delta}\left(t_{m_{k}}, x_{m_{k}}, \phi\right)+I_{i j}^{2, \delta}\left(t_{m_{k}}, x_{m_{k}}, \bar{u}^{i j, m_{k}}\right)\right] .
\end{gathered}
$$

From which we deduce, in dividing both hand-sides of (4.22) by $m_{k}$ and then taking the limit as $k \rightarrow \infty$, that

$$
\epsilon_{k}=\left(\bar{u}^{i j, m_{k}}\left(t_{m_{k}}, x_{m_{k}}\right)-\min _{l \in A_{2}^{j}}\left(\bar{u}^{i l, m_{k}}\left(t_{m_{k}}, x_{m_{k}}\right)+\bar{g}_{j l}\left(t_{m_{k}}, x_{m_{k}}\right)\right)\right)^{+} \rightarrow_{k} 0 .
$$

Next fix $k_{0}$ and let $k \geq k_{0}$. As the sequence $\left(\bar{u}^{i j, m}\right)_{m}$ is decreasing then

$$
\begin{aligned}
\bar{u}^{i j, m_{k}}\left(t_{m_{k}}, x_{m_{k}}\right) & \leq \min _{l \in A_{2}^{j}}\left(\bar{u}^{i l, m_{k}}\left(t_{m_{k}}, x_{m_{k}}\right)+\bar{g}_{j l}\left(t_{m_{k}}, x_{m_{k}}\right)\right)+\epsilon_{k} \\
& \leq \min _{l \in A_{2}^{j}}\left(\bar{u}^{i l, m_{k_{0}}}\left(t_{m_{k}}, x_{m_{k}}\right)+\bar{g}_{j l}\left(t_{m_{k}}, x_{m_{k}}\right)\right)+\epsilon_{k}
\end{aligned}
$$

Take the limit w.r.t $k$, using continuity of $\bar{u}^{i l, m_{k_{0}}}$ then send $k_{0}$ to $+\infty$ to obtain:

$$
\bar{u}^{i j}(t, x) \leq \min _{l \in A_{2}^{j}}\left(\bar{u}^{i l}(t, x)+\bar{g}_{j l}(t, x)\right)
$$

Next there exists a subsequence of $\left\{m_{k}\right\}$ (which we still denote by $\left\{m_{k}\right\}$ ) such that: 
(i) $\forall(p, l) \in A_{i}^{1} \times A_{j}^{2},\left(\bar{u}^{p l, m_{k}}\left(t_{m_{k}}, x_{m_{k}}\right)\right)_{k}$ is convergent and then $\lim _{k} \bar{u}^{p l, m_{k}}\left(t_{m_{k}}, x_{m_{k}}\right) \leq \bar{u}^{p l}(t, x)$;

(ii) $I_{\delta}^{1}\left(t_{m_{k}}, x_{m_{k}}, \phi\right) \rightarrow_{k} I_{\delta}^{1}(t, x, \phi)$ and $\left.I_{i j}^{1, \delta}\left(t_{m_{k}}, x_{m_{k}}, \phi\right)\right) \rightarrow_{k} I_{i j}^{1, \delta}(t, x, \phi)$;

(iii) By Fatou's Lemma, $\lim \sup _{k} I_{\delta}^{2}\left(t_{m_{k}}, x_{m_{k}}, D_{x} \phi\left(t_{m_{k}}, x_{m_{k}}\right), \bar{u}^{i j, m_{k}}\right) \leq I_{\delta}^{2}\left(t, x, D_{x} \phi(t, x), \bar{u}^{i j}\right)$ and $\left.\left.\lim \sup _{k} I_{i j}^{2, \delta}\left(t_{m_{k}}, x_{m_{k}}, \bar{u}^{i j, m_{k}}\right)\right)\right) \leq I_{i j}^{2, \delta}\left(t, x, \bar{u}^{i j}\right)$.

Let us now set:

$$
\begin{aligned}
& \Delta_{k}:=g^{i j}\left[t_{m_{k}}, x_{m_{k}},\left(\bar{u}^{p l, m_{k}}\left(t_{m_{k}}, x_{m_{k}}\right)\right)_{(p, l) \in A^{1} \times A^{2}}, \sigma\left(t_{m_{k}}, x_{m_{k}}\right)^{\top} D_{x} \phi\left(t_{m_{k}}, x_{m_{k}}\right),\right. \\
& \left.I_{i j}^{1, \delta}\left(t_{m_{k}}, x_{m_{k}}, \phi\right)+I_{i j}^{2, \delta}\left(t_{m_{k}}, x_{m_{k}}, \bar{u}^{i j, m_{k}}\right)\right] \\
& -g^{i j}\left[t_{m_{k}}, x_{m_{k}},\left(\bar{u}^{p l, m_{k}}\left(t_{m_{k}}, x_{m_{k}}\right)\right)_{(p l) \in A^{1} \times A^{2}}, \sigma\left(t_{m_{k}}, x_{m_{k}}\right)^{\top} D_{x} \phi\left(t_{m_{k}}, x_{m_{k}}\right), I_{i j}^{1, \delta}(t, x, \phi)+I_{i j}^{2, \delta}\left(t, x, \bar{u}^{i j}\right)\right]
\end{aligned}
$$

Then, by linearizing $g^{i j}$ w.r.t. $q$, there exists a non-negative bounded quantity $\Xi_{2}$ such that

$$
\begin{aligned}
\Delta_{k} & =\Xi_{2} \times\left(I_{i j}^{1, \delta}\left(t_{m_{k}}, x_{m_{k}}, \phi\right)+I_{i j}^{2, \delta}\left(t_{m_{k}}, x_{m_{k}}, \bar{u}^{i j, m_{k}}\right)-I_{i j}^{1, \delta}(t, x, \phi)-I_{i j}^{2, \delta}\left(t, x, \bar{u}^{i j}\right)\right. \\
& \leq C_{i j} \times\left(I_{i j}^{1, \delta}\left(t_{m_{k}}, x_{m_{k}}, \phi\right)+I_{i j}^{2, \delta}\left(t_{m_{k}}, x_{m_{k}}, \bar{u}^{i j, m_{k}}\right)-I_{i j}^{1, \delta}(t, x, \phi)-I_{i j}^{2, \delta}\left(t, x, \bar{u}^{i j}\right)\right)^{+}
\end{aligned}
$$

where $C_{i j}$ is the Lipschitz constant of $g^{i j}$. Therefore, with (ii)-(iii) above, we have that $\lim \sup _{k} \Delta_{k} \leq 0$.

Going back now to (4.22), and take the limit superior w.r.t. $k$ to get:

$$
\begin{aligned}
-\partial_{t} \phi(t, x)-\overline{\mathcal{L}} \phi(t, x) & \leq I_{\delta}^{1}(t, x, \phi)+I_{\delta}^{2}\left(t, x, D_{x} \phi(t, x), \bar{u}^{i j}\right)+ \\
& g^{i j}\left[t, x,\left(\bar{u}^{p l}(t, x)\right)_{(p, l) \in \Gamma}, \sigma(t, x)^{\top} D_{x} \phi(t, x), I_{i j}^{1, \delta}(t, x, \phi)+I_{i j}^{2, \delta}\left(t, x, \bar{u}^{i j}\right)\right] .
\end{aligned}
$$

But $\bar{u}^{i j}(t, x)=\phi(t, x)$ and $\bar{u}^{i j} \leq \phi$, then $I_{\delta}^{2}\left(t, x, D_{x} \phi(t, x), \bar{u}^{i j}\right) \leq I_{\delta}^{2}\left(t, x, D_{x} \phi(t, x), \phi\right)$ and $I_{i j}^{2, \delta}\left(t, x, \bar{u}^{i j}\right) \leq$ $I_{i j}^{2, \delta}(t, x, \phi)$. Plugging now this inequality in the previous one to obtain

$$
-\partial_{t} \phi(t, x)-\overline{\mathcal{L}} \phi(t, x)-I(t, x, \phi)-g^{i j}\left[t, x,\left(\bar{u}^{p l}(t, x)\right)_{(p, l) \in \Gamma}, \sigma(t, x)^{\top} D_{x} \bar{u}^{i j}(t, x), I_{i j}(t, x, \phi)\right] \leq 0 .
$$

Therefore $\bar{u}^{i j}$ is a viscosity subsolution of

$$
\left\{\begin{array}{l}
\min \left\{\left(w-L^{i j}\left[\left(\bar{u}^{k l}\right)_{(k, l) \in \Gamma}\right]\right)(t, x) ; \max \left\{\left(w-U^{i j}\left[\left(\bar{u}^{k l}\right)_{(k, l) \in \Gamma}\right]\right)(t, x) ;\right.\right. \\
\left.\left.-\partial_{t} w(t, x)-\mathcal{L} w(t, x)-g^{i j}\left(t, x,\left[\left(\bar{u}^{p l}(t, x)\right)_{(p, l) \in \Gamma^{-(i, j)}}, w\right], \sigma(t, x)^{\top} D_{x} w(t, x), I_{i j}(t, x, w)\right)\right\}\right\}=0 ; \\
w(T, x)=h^{i j}(x) .
\end{array}\right.
$$

As $(i, j)$ in $\Gamma$ is arbitrary then $\left(\bar{u}^{i j}\right)_{(i, j) \in A^{1} \times A^{2}}$ is a viscosity subsolution of $(2.1)$.

Proposition 4.5. Let $m_{0}$ be fixed in $\mathbf{N}$. Then the family $\left(\bar{u}^{i j, m_{0}}\right)_{(i, j) \in A^{1} \times A^{2}}$ is a viscosity supersolution of system (2.1).

Proof. Recall that $\left(\bar{Y}^{i j, m_{0}}, \bar{Z}^{i j, m_{0}}, \bar{U}^{i j, m_{0}}, \bar{K}^{i j, m_{0}}\right)_{(i, j) \in A^{1} \times A^{2}}$ solves the system of reflected BSDEs (4.6). Therefore if we set $\bar{K}_{s}^{i j, m_{0},-}:=m_{0} \int_{0}^{s}\left(Y_{r}^{i j, m_{0}}-\min _{l \in A_{j}^{2}}\left(Y_{r}^{i l, m_{0}}+\bar{g}_{j l}\left(r, X_{r}^{t, x}\right)\right)\right)^{+} d r, s \leq T$, then $\left(\bar{Y}^{i j, m_{0}}, \bar{Z}^{i j, m_{0}}, \bar{U}^{i j, m_{0}}, \bar{K}^{i j, m_{0}}, \bar{K}^{i j, m_{0},-}\right)_{(i, j) \in A^{1} \times A^{2}}$ is a solution of the following system of reflected BSDEs 
with bilateral interconnected obstacles: for any $(i, j) \in \Gamma$ and $s \leq T$,

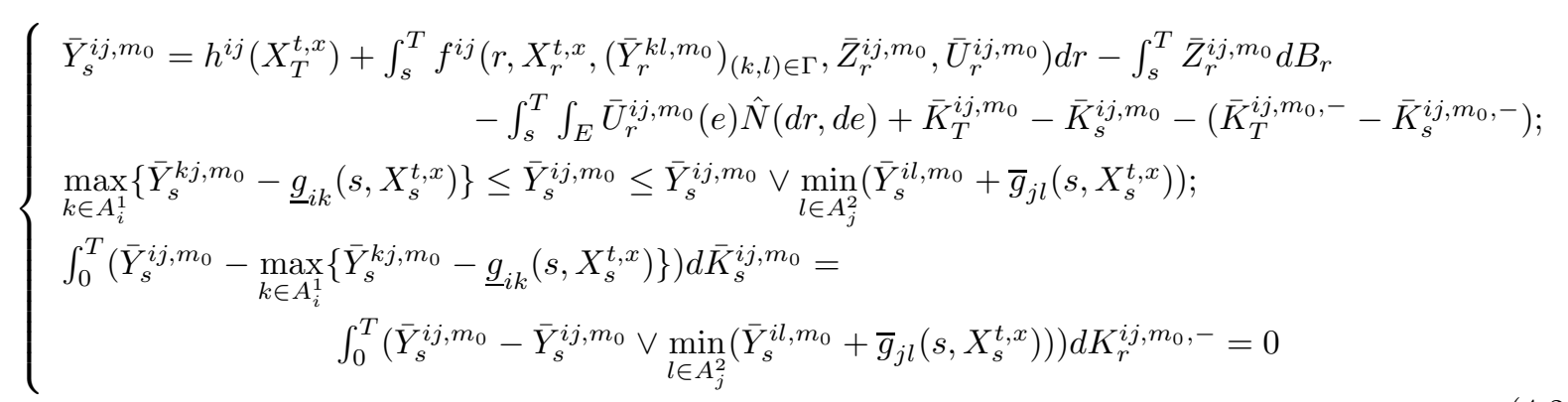

On the other hand we know by (4.9) that there exist deterministic continuous functions $\left(\bar{u}^{i j, m_{0}}\right)_{(i, j) \in A^{1} \times A^{2}}$ in $\Pi_{g}$ such that, for every $t \leq T$,

$$
\bar{Y}_{s}^{i j, m_{0}}=\bar{u}^{i j, m_{0}}\left(s, X_{s}^{t, x}\right), s \in[t, T] .
$$

Then using a result by Harraj et al. [21] we deduce that for any $(i, j) \in \Gamma, \bar{u}^{i j, m_{0}}$ is a viscosity solution of the following IPDE:

$$
\left\{\begin{array}{l}
\min \left\{\bar{u}^{i j, m_{0}}(t, x)-\max _{k \in A_{i}^{1}}\left\{\bar{u}^{k j, m_{0}}(t, x)-\underline{g}_{i k}(t, x)\right\} ;\right. \\
\max \left\{\bar{u}^{i j, m_{0}}(t, x)-\bar{u}^{i j, m_{0}}(t, x) \vee \min _{l \in A_{j}^{2}}\left(\bar{u}^{i l, m_{0}}(t, x)+\bar{g}_{j l}(t, x)\right) ;-\partial_{t} \bar{u}^{i j, m_{0}}(t, x)-\mathcal{L} \bar{u}^{i j, m_{0}}(t, x)\right. \\
\left.\left.\quad-g^{i j}\left(t, x,\left(\bar{u}^{k l, m_{0}}(t, x)\right)_{(k, l) \in A^{1} \times A^{2}}, \sigma(t, x)^{\top} D_{x} \bar{u}^{i j, m_{0}}(t, x), I_{i j}\left(t, x, \bar{u}^{i j, m_{0}}\right)\right)\right\}\right\}=0 \\
\bar{u}^{i j, m_{0}}(T, x)=h^{i j}(x) .
\end{array}\right.
$$

But

$$
\bar{u}^{i j, m_{0}}(t, x)-\bar{u}^{i j, m_{0}}(t, x) \vee \min _{l \in A_{j}^{2}}\left(\bar{u}^{i l, m_{0}}(t, x)+\bar{g}_{j l}(t, x)\right) \leq \bar{u}^{i j, m_{0}}(t, x)-\min _{l \in A_{j}^{2}}\left(\bar{u}^{i l, m_{0}}(t, x)+\bar{g}_{j l}(t, x)\right)
$$

Therefore $\bar{u}^{i j, m_{0}}$ is a supersolution of

$$
\left\{\begin{array}{l}
\min \left\{\bar{u}^{i j, m_{0}}(t, x)-\max _{k \in A_{i}^{1}}\left\{\bar{u}^{k j, m_{0}}(t, x)-\underline{g}_{i k}(t, x)\right\} ;\right. \\
\max \left\{\bar{u}^{i j, m_{0}}(t, x)-\min _{l \in A_{j}^{2}}\left(\bar{u}^{i l, m_{0}}(t, x)+\bar{g}_{j l}(t, x)\right) ;-\partial_{t} \bar{u}^{i j, m_{0}}(t, x)-\mathcal{L} \bar{u}^{i j, m_{0}}(t, x)\right. \\
\left.\left.\quad-g^{i j}\left(t, x,\left(\bar{u}^{k l, m_{0}}(t, x)\right)_{(k, l) \in A^{1} \times A^{2}}, \sigma(t, x)^{\top} D_{x} \bar{u}^{i j, m_{0}}(t, x), I_{i j}\left(t, x, \bar{u}^{i j, m_{0}}\right)\right)\right\}\right\}=0 ; \\
\bar{u}^{i j, m_{0}}(T, x)=h^{i j}(x) .
\end{array}\right.
$$

As $(i, j)$ is arbiratry then $\left(\bar{u}^{i j, m_{0}}\right)_{(i, j) \in A^{1} \times A^{2}}$ is a viscosity supersolution of system $(2.1)$.

Consider now the set $\mathcal{U}_{m_{0}}$ defined as follows:

$\mathcal{U}_{m_{0}}=\left\{\vec{u}:=\left(u^{i j}\right)_{(i, j) \in A^{1} \times A^{2}}\right.$ s.t. $\vec{u}$ is a subsolution of $(2.1)$ and $\left.\forall(i, j) \in A^{1} \times A^{2}, \bar{u}^{i j} \leq u^{i j} \leq \bar{u}^{i j, m_{0}}\right\}$.

$\mathcal{U}_{m_{0}}$ is not empty since it contains $\left(\bar{u}^{i j}\right)_{(i, j) \in A^{1} \times A^{2}}$. Next for $(t, x) \in[0, T] \times \mathbf{R}^{k}$ and $(i, j) \in \Gamma$, let us set:

$$
{ }^{m_{0}} u^{i j}(t, x)=\sup \left\{u^{i j}(t, x),\left(u^{k l}\right)_{(k, l) \in A^{1} \times A^{2}} \in \mathcal{U}_{m_{0}}\right\} .
$$

We are now ready to give the main result of this paper: 
Theorem 4.1. The family $\left({ }^{m_{0}} u^{i j}\right)_{(i, j) \in A^{1} \times A^{2}}$ does not depend on $m_{0}$ and is the unique continuous viscosity solution in the class $\Pi_{g}$ of the system (2.1). Moreover ${ }^{m_{0}} u^{i j}=\bar{u}^{i j}$ for any $(i, j) \in A^{1} \times A^{2}$.

Proof. Firs note that w.l.o.g we assume that for any $(i, j) \in A^{1} \times A^{2}$, the function $y \in \mathbf{R} \mapsto g_{i j}\left(t, x,\left[\left(y^{k l}\right)_{(k, l) \in \Gamma^{-(i, j)}}, y\right], z, q\right)$ is also non-decreasing when the other variables are fixed.

To begin with, note that for any $(i, j) \in A^{1} \times A^{2}, \bar{u}^{i j} \leq m_{0} u^{i j} \leq \bar{u}^{i j, m_{0}}$. Since $\bar{u}^{i j}$ and $\bar{u}^{i j, m_{0}}$ are of polynomial growth, then $\left({ }^{m_{0}} u^{i j}\right)_{(i, j) \in A^{1} \times A^{2}}$ belongs also to $\Pi_{g}$. The remaining of the proof is divided into two steps and for ease of notation, we denote $\left({ }^{m_{0}} u^{i j}\right)_{(i, j) \in \Gamma}$ simply by $\left(u^{i j}\right)_{(i, j) \in \Gamma}$ as no confusion is possible.

Step 1: First we show that $\left(u^{i j}\right)_{(i, j) \in A^{1} \times A^{2}}$ is a subsolution of (2.1). As $\bar{u}^{i j} \leq u^{i j} \leq \bar{u}^{i j, m_{0}}$ then $\bar{u}^{i j} \leq u^{i j, *} \leq \bar{u}^{i j, m_{0}}$ since $\bar{u}^{i j}$ is $u s c$ and $\bar{u}^{i j, m_{0}}$ is continuous. Therefore, for any $x \in \mathbf{R}^{k}$, since $\bar{u}^{i j}(T, x)=$ $\bar{u}^{i j, m_{0}}(T, x)=h^{i j}(x)$, we have $u^{i j, *}(T, x)=h^{i j}(x)$.

Next let $\left(\tilde{u}^{i j}\right)_{(i, j) \in A^{1} \times A^{2}}$ be an arbitrary element of $\mathcal{U}_{m_{0}}$ and let $(i, j)$ be fixed. Let $(t, x) \in(0, T) \times \mathbf{R}^{k}$ and $\phi \in \mathcal{C}^{1,2}\left([0, T] \times \mathbf{R}^{k}\right)$ such that $\tilde{u}^{i j, *}(t, x)=\phi(t, x)$ and $\tilde{u}^{i j, *} \leq \phi$. Then

$$
\begin{aligned}
\min \left\{\left(\tilde{u}^{i j, *}-L^{i j}\left[\left(\tilde{u}^{k l, *}\right)_{\left.\left.(k, l) \in A^{1} \times A^{2}\right]\right)(t, x) ; \max \left\{\left(\tilde{u}^{i j, *}-U^{i j}\left[\left(\tilde{u}^{k l, *}\right)_{\left.(k, l) \in A^{1} \times A^{2}\right]}\right)(t, x) ;\right.\right.}\right.\right.\right. \\
\left.\left.\quad-\partial_{t} \phi(t, x)-\mathcal{L} \phi(t, x)-g^{i j}\left(t, x,\left(\tilde{u}^{k l, *}(t, x)\right)_{(k, l) \in A^{1} \times A^{2}}, \sigma(t, x)^{\top} D_{x} \phi(t, x), I_{i j}(t, x, \phi)\right)\right\}\right\} \leq 0 .
\end{aligned}
$$

By definition, for any $(k, l) \in \Gamma, \tilde{u}^{k l} \leq u^{k l}$ and then $\tilde{u}^{k l, *} \leq u^{k l, *}$. Using now the monotonicity property (A2), we obtain

$$
\begin{aligned}
& \min \left\{\left(\tilde{u}^{i j, *}-L^{i j}\left[\left(u^{k l, *}\right)_{(k, l) \in A^{1} \times A^{2}}\right]\right)(t, x) ; \max \left\{\left(\tilde{u}^{i j, *}-U^{i j}\left[\left(u^{k l, *}\right)_{\left.\left.(k, l) \in A^{1} \times A^{2}\right]\right)(t, x) ;}\right.\right.\right.\right. \\
& \left.\left.\quad-\partial_{t} \phi(t, x)-\mathcal{L} \phi(t, x)-g^{i j}\left(t, x,\left[\left(u^{k l, *}\right)_{(k, l) \in \Gamma^{-(i, j)}}, \tilde{u}^{i j, *}\right](t, x), \sigma(t, x)^{\top} D_{x} \phi(t, x), I_{i j}(t, x, \phi)\right)\right\}\right\} \leq 0 .
\end{aligned}
$$

This means that $\tilde{u}^{i j}$ is a subsolution of the following equation:

$$
\left\{\begin{array}{l}
\min \left\{\left(w-L^{i j}\left[\left(u^{k l, *}\right)_{\left.\left.(k, l) \in A^{1} \times A^{2}\right]\right)(t, x) ; \max \left\{\left(w-U^{i j}\left[\left(u^{k l, *}\right)_{\left.\left.(k, l) \in A^{1} \times A^{2}\right]\right)(t, x) ;}\right.\right.\right.}-\partial_{t} w(t, x)-\mathcal{L} w(t, x)-g^{i j}\left(t, x,\left[\left(u^{k l, *}\right)_{(k, l) \in \Gamma^{-(i, j)}}, w\right](t, x), \sigma(t, x)^{\top} D_{x} w(t, x), I_{i j}(t, x, w)\right)\right\}\right\}=0 ;\right. \\
w(T, x)=h^{i j}(x) .
\end{array}\right.
$$

Consequently, by a result by Barles-Imbert ([4], Theorem 2, pp.577), $u^{i j}$ is a subsolution of

$$
\left\{\begin{array}{l}
\min \left\{\left(w-L^{i j}\left[\left(u^{k l, *}\right)_{\left.\left.(k, l) \in A^{1} \times A^{2}\right]\right)(t, x) ; \max \left\{\left(w-U^{i j}\left[\left(u^{k l, *}\right)_{\left.\left.(k, l) \in A^{1} \times A^{2}\right]\right)(t, x) ;}\right.\right.\right.}-\partial_{t} w(t, x)-\mathcal{L} w(t, x)-g^{i j}\left(t, x,\left[\left(u^{k l, *}\right)_{(k, l) \in \Gamma^{-(i, j)}}, w\right](t, x), \sigma(t, x)^{\top} D_{x} w(t, x), I_{i j}(t, x, w)\right)\right\}\right\}=0 ;\right. \\
w(T, x)=h^{i j}(x) .
\end{array}\right.
$$

As $(i, j)$ in $\Gamma$ is arbitrary then $\left(u^{i j}\right)_{(i, j) \in A^{1} \times A^{2}}$ is a subsolution of $(2.1)$.

Step 2: We will now show, by contradiction, that $\left(u^{i j}\right)_{(i, j) \in A^{1} \times A^{2}}$ is a supersolution of (2.1). First note that for any $(i, j) \in A^{1} \times A^{2}, \underline{u}^{i j}=\underline{u}_{*}^{i j} \leq \bar{u}_{*}^{i j} \leq u_{*}^{i j} \leq \bar{u}_{*}^{i j, m_{0}}=\bar{u}^{i j, m_{0}}$, since $\bar{u}^{i j, m_{0}}$ is continuous and $\underline{u}^{i j}$ is lsc. Therefore, for any $x \in \mathbf{R}^{k}$, since $\underline{u}^{i j}(T, x)=h^{i j}(x)=\bar{u}^{i j, m_{0}}(T, x)$, it holds, $u_{*}^{i j}(T, x)=h^{i j}(x)$. 
The rest of the proof is rather classical and can be read e.g. in [5] up to some adaptations. However we give it for completeness. So suppose that there exist $(i, j) \in A^{1} \times A^{2},(t, x) \in(0, T) \times \mathbf{R}^{k}$ and a $\mathcal{C}^{1,2}$-function $\phi$ such that $u_{*}^{i j}(t, x)=\phi(t, x), u_{*}^{i j}(s, y)>\phi(s, y)$ in $(0, T) \times \mathbf{R}^{k}-\{(t, x)\}$ and

$$
\begin{aligned}
\min & \left\{\left(\phi-L^{i j}\left[\left(u_{*}^{k l}\right)_{\left.\left.(k, l) \in A^{1} \times A^{2}\right]\right)(t, x) ; \max \left\{\left(\phi-U^{i j}\left[\left(u_{*}^{k l}\right)_{\left.(k, l) \in A^{1} \times A^{2}\right]}\right]\right)(t, x) ;\right.}\right.\right.\right. \\
& \left.\left.-\partial_{t} \phi(t, x)-\mathcal{L} \phi(t, x)-g^{i j}\left(t, x,\left[\left(u_{*}^{k l}\right)_{(k, l) \in \Gamma^{-(i, j)}}, \phi\right](t, x), \sigma(t, x)^{\top} D_{x} \phi(t, x), I_{i j}(t, x, \phi)\right)\right\}\right\}<0 .
\end{aligned}
$$

Then by continuity of the equation, continuity and monotonicity of $g^{i j}$ and lower semi-continuity of $u_{*}^{k l}$, there exist two constants $\epsilon_{1}>0$ and $\delta_{1}>0$ such that: $\forall(s, y) \in B\left((t, x), \delta_{1}\right)$ and $0 \leq \epsilon \leq \epsilon_{1}$ we have:

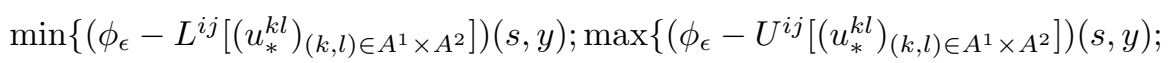

$$
\begin{aligned}
& \left.\left.-\partial_{t} \phi_{\epsilon}(s, y)-\mathcal{L} \phi_{\epsilon}(s, y)-g^{i j}\left(s, y,\left[\left(u_{*}^{k l}\right)_{(k, l) \in \Gamma^{-(i, j)}}, \phi_{\epsilon}\right](s, y), \sigma(s, y)^{\top} D_{x} \phi_{\epsilon}(s, y), I_{i j}\left(s, y, \phi_{\epsilon}\right)\right)\right\}\right\} \leq 0
\end{aligned}
$$

where $\phi_{\epsilon}=\phi+\epsilon$. Next since $(t, x)$ is a strict minimum of $u_{*}^{i j}-\phi$, there are constants $0<\epsilon_{2}$ and $0<\delta_{2} \leq \delta_{1}$ such that $u_{*}^{i j}-\phi>\epsilon_{2}$ on $\partial B\left((t, x), \delta_{2}\right)$. Now let us set $\epsilon_{3}=\min \left(\epsilon_{1}, \epsilon_{2}\right)$ and let us define $\left(w^{k l}\right)_{(k, l) \in A^{1} \times A^{2}}$ as follows:

$$
w^{k l}=u^{k l, *} \text { if }(k, l) \neq(i, j) \text { and } w^{i j}=\left\{\begin{array}{l}
\max \left(\phi+\epsilon_{3}, u^{i j, *}\right) \text { on } B\left((t, x), \delta_{2}\right) \subset(0, T) \times \mathbf{R}^{k} \\
u^{i j, *} \text { elsewhere }
\end{array}\right.
$$

Then $\left(w^{k l}\right)_{(k, l) \in A^{1} \times A^{2}}$ belongs to $\Pi_{g}$ and is a viscosity subsolution of (2.1). Indeed, first note that for any $(k, l) \in \Gamma, w^{k l}$ is usc and $w^{k l}(T, x)=u^{k l, *}(T, x)=h^{k l}(x)$. Next let $(s, y) \in(0, T) \times \mathbf{R}^{k}$. If $(s, y)$ does not belong to $B\left((t, x), \delta_{2}\right)$ then the subsolution property stems from the one of $\left(u^{k l, *}\right)_{(k, l) \in A^{1} \times A^{2} \text {. Assume now }}$ that $(s, y) \in B\left((t, x), \delta_{2}\right)$. If $(k, l) \neq(i, j)$, then the subsolution property stems from the one of $u^{k l, *}$, in taking into account of $w^{i j} \geq u^{i j, *}, w^{k_{1} l_{1}}=u^{k_{1} l_{1}, *}$ if $\left(k_{1}, l_{1}\right) \neq(i, j)$ and monotonicity of $g^{k l}$. Finally let us deal with the case when $(k, l)=(i, j)$. Let $\psi$ be a $\mathcal{C}^{1,2}$-function such that $w^{i j}(s, y)=\psi(s, y)$ and $\psi-w^{i j}$ has a strict global minimum in $(s, y) \in(0, T) \times \mathbf{R}^{k}$. If $w^{i j}(s, y)=u^{i j, *}(s, y)$ then

$$
\begin{aligned}
\min \{ & \left(\psi-L^{i j}\left[\left(u^{k l, *}\right)_{\left.(k, l) \in A^{1} \times A^{2}\right]}\right](s, y) ; \max \left\{\left(\psi-U^{i j}\left[\left(u^{k l, *}\right)_{(k, l) \in A^{1} \times A^{2}}\right]\right)(s, y) ;\right.\right. \\
& \left.\left.-\partial_{t} \psi(t, x)-\mathcal{L} \psi(s, y)-g^{i j}\left(s, y,\left[\left(u^{k l, *}\right)_{(k, l) \in \Gamma^{-(i, j)}}, \psi\right](s, y), \sigma(s, y)^{\top} D_{x} \psi(s, y), I_{i j}(s, y, \psi)\right)\right\}\right\} \leq 0
\end{aligned}
$$

since $w^{i j} \geq u^{i j, *}$ and then $\psi-u^{i j, *}$ has a strict global minimum in $(s, y),\left(\psi-u^{i j, *}\right)(s, y)=0$ and $\left(u^{k l, *}\right)_{(k, l) \in A^{1} \times A^{2}}$ is a subsolution of system (2.1). Thus,

$$
\begin{aligned}
\min \{ & \left(\psi-L^{i j}\left[\left(w^{k l, *}\right)_{\left.(k, l) \in A^{1} \times A^{2}\right]}\right](s, y) ; \max \left\{\left(\psi-U^{i j}\left[\left(w^{k l, *}\right)_{\left.(k, l) \in A^{1} \times A^{2}\right]}\right](s, y) ;\right.\right.\right. \\
& \left.\left.-\partial_{t} \psi(t, x)-\mathcal{L} \psi(s, y)-g^{i j}\left(s, y,\left[\left(w^{k l, *}\right)_{(k, l) \in \Gamma^{-(i, j)}}, \psi\right](s, y), \sigma(s, y)^{\top} D_{x} \psi(s, y), I_{i j}(s, y, \psi)\right)\right\}\right\} \leq 0 .
\end{aligned}
$$

Finally if $w^{i j}(s, y)=\phi(s, y)+\epsilon_{3}$ then $\phi(s, y)+\epsilon_{3}=\psi(s, y)$ and $\phi+\epsilon_{3} \leq \psi$ on $B\left((t, x), \delta_{2}\right)$. It implies that

$$
\partial_{t} \phi(s, y)=\partial_{t} \psi(s, y), D_{x} \phi(s, y)=D_{x} \psi(s, y) \text { and } D_{x x}^{2} \phi(s, y) \leq D_{x x}^{2} \psi(s, y)
$$

and by (4.24) we deduce that $w^{i j}$ verifies (4.25). Therefore $w^{i j}$ satisfies the subsolution property and $\left(w^{k l}\right)_{(k, l) \in A^{1} \times A^{2}}$ is a viscosity subsolution of (2.1). But we have,

$$
w_{*}^{i j}(t, x) \geq \max \left\{\phi(t, x)+\epsilon_{3}, u_{*}^{i j}(t, x)\right\}=\phi(t, x)+\epsilon_{3}=u_{*}^{i j}(t, x)+\epsilon_{3} .
$$


Thus there exists $\left(t_{0}, x_{0}\right) \in(0, T) \times \mathbf{R}^{k}$ such that $w^{i j}\left(t_{0}, x_{0}\right)>u^{i j}\left(t_{0}, x_{0}\right)$. But this is contradictory to the definition of $u^{i j}$. Therefore $\left(u^{k l}\right)_{(k, l) \in A^{1} \times A^{2}}$ is a supersolution of (2.1) and the proof is complete.

Now, by Corollary $(3.1),\left({ }^{m_{0}} u^{i j}\right)_{(i, j) \in A^{1} \times A^{2}}$ (i.e. $\left.\left(u^{i j}\right)_{(i, j) \in A^{1} \times A^{2}}\right)$ does not depend on $m_{0}$ since the solution of $(2.1)$ is the unique. On the other hand for any $(i, j)$, we have

$$
\bar{u}^{i j} \leq u^{i j}:={ }^{m_{0}} u^{i j} \leq u^{i j, m_{0}}
$$

and in taking the limit wrt $m_{0}$ we obtain $\bar{u}^{i j}=u^{i j}$, for any $(i, j) \in A_{1} \times A_{2}$.

As a by-product of the above construction we have the following result related to the limit of the increasing scheme:

Theorem 4.2. The family $\left(\underline{u}^{i j}\right)_{(i, j) \in A^{1} \times A^{2}}$ is continuous and of polynomial growth and is the unique viscosity solution in $\Pi_{g}$ of the max-min problem, i.e., for any $(i, j) \in \Gamma$,

$$
\left\{\begin{array}{l}
\max \left\{\left(v^{i j}-U^{i j}[\vec{v}]\right)(t, x) ; \min \left\{\left(v^{i j}-L^{i j}[\vec{v}]\right)(t, x) ;\right.\right. \\
\left.\left.\quad-\partial_{t} v^{i j}(t, x)-\mathcal{L} v^{i j}(t, x)-g^{i j}\left(t, x,\left(v^{k l}(t, x)\right)_{(k, l) \in A^{1} \times A^{2}}, \sigma(t, x)^{\top} D_{x} v^{i j}(t, x), I^{i j}\left(t, x, v^{i j}\right)\right)\right\}\right\}=0 ; \\
v^{i j}(T, x)=h^{i j}(x) .
\end{array}\right.
$$

Proof. Actually in considering the opposite of the increasing scheme defined in (4.14), which becomes a decreasing one, we obtain that $\left(-\underline{u}^{i j}\right)_{(i, j) \in A^{1} \times A^{2}}$ is continuous and of polynomial growth and is the unique viscosity solution in $\Pi_{g}$ of the following system: $\forall(i, j) \in A^{1} \times A^{2}$,

$$
\left\{\begin{array}{l}
\min \left\{\left(\underline{\mathrm{v}}^{i j}-\max _{k \in A_{j}^{2}}\left\{\underline{\mathrm{v}}^{i k}-\bar{g}_{k j}\right\}\right)(t, x) ; \max \left\{\left(\underline{\mathrm{v}}^{i j}-\min _{l \in A_{i}^{1}}\left\{\underline{\mathrm{v}}^{l j}+\underline{g}_{i l}\right\}\right)(t, x) ;\right.\right. \\
\left.\left.-\partial_{t} \underline{\mathrm{v}}^{i j}(t, x)-\mathcal{L}_{\underline{\mathrm{v}}}^{i j}(t, x)+g^{i j}\left(t, x,\left(-\underline{\mathrm{v}}^{k l}(t, x)\right)_{(k, l) \in A^{1} \times A^{2}}, \sigma(t, x)^{\top} D_{x}\left(-\underline{\mathrm{v}}^{i j}\right)(t, x),-I_{i j}\left(t, x, \underline{\mathrm{v}}^{i j}\right)\right)\right\}\right\}=0 ; \\
\underline{\mathrm{v}}^{i j}(T, x)=-h^{i j}(x) .
\end{array}\right.
$$

Using now a result by G.Barles ([2], pp.18) we obtain that $\left(\underline{u}^{i j}\right)_{(i, j) \in A^{1} \times A^{2}}$ is the unique viscosity solution in $\Pi_{g}$ of $(4.26)$.

\section{References}

[1] Alvarez, O., Tourin, A. (1996). Viscosity solutions of non-linear integro-differential equations. Annales de l'Institut Henri Poincaré, Non Linear Analysis, 13(3), 293-317.

[2] Barles, G. (1994). Solutions de viscosité des équations de Hamilton-Jacobi. Mathématiques \& Applications, 17, Springer-Verlag, Paris.

[3] Barles, G., Buckdahn, R., Pardoux, E. (1997). Backward stochastic differential equations and integralpartial differential equations. Stochastics: An International Journal of Probability and Stochastic Processes (Vol. 60, No. 1-2, pp. 57-83). 
[4] Barles, G., Imbert, C. (2007). Second-order elliptic integro-differential equations: viscosity solutions' theory revisited. Annales de l'Institut Henri Poincaré, Non Linear Analysis, 25(3), 567-585.

[5] Biswas, I. H., Jakobsen, E. R., Karlsen, K. H. (2010). Viscosity solutions for a system of integro-PDEs and connections to optimal switching and control of jump-diffusion processes. Applied Mathematics \& Optimization, Vol. 62, No. 1, pp. 47-80.

[6] Chassagneux, J. F., Elie, R., Kharroubi, I. (2011). A note on existence and uniqueness for solutions of multidimensional reflected BSDEs. Electronic Communications in Probability, 16, 120-128.

[7] Crandall, M. G., Ishii, H., Lions, P. L. (1992). User's guide to viscosity solutions of second order partial differential equations. Bulletin of the American Mathematical Society, 27(1), 1-67.

[8] Dellacherie, C., Meyer, P. A. (1980). Probabilités et Potentiel: Chapitres V à VIII. Hermann. Paris.

[9] Djehiche, B., Hamadene, S., Morlais, M. A. (2015). Viscosity solutions of systems of variational inequalities with interconnected bilateral obstacles. Funkcialaj Ekvacioj, 58(1), 135-175.

[10] Djehiche, B. Hamadene, S., Morlais, M.-A., Zhao X. (2017). On the Equality of Solutions of Max-Min and Min-Max Systems of Variational Inequalities with Interconnected Bilateral Obstacles. J. Math. Anal. Appl. 452 (2017), no. 1, 148-175.

[11] Djehiche, B., Hamadene, S., Popier, A. (2009). A finite horizon optimal multiple switching problem. SIAM Journal on Control and Optimization, 48(4), 2751-2770.

[12] El Asri, B., Hamadene, S. (2009). The finite horizon optimal multi-modes switching problem: the viscosity solution approach. Applied Mathematics \& Optimization, 60(2), 213-235.

[13] Elie, R., Kharroubi, I. (2009). Constrained backward SDEs with jumps: Application to optimal switching. Preprint.

[14] Essaky, E. H. (2008). Reflected backward stochastic differential equation with jumps and RCLL obstacle. Bulletin des Sciences Mathématiques, 132(8), 690-710.

[15] Fujiwara, T., , Kunita, H. (1985). Stochastic differential equations of jump type and Lévy processes in diffeomorphisms group. Journal of mathematics of Kyoto University, 25(1), 71-106.

[16] Hamadene, S., Hdhiri, I. (2007). The stopping and starting problem in the model with jumps. PAMM, $7(1), 1081803-1081804$.

[17] Hamadene, S., Jeanblanc, M. (2007). On the starting and stopping problem: application in reversible investments. Mathematics of Operations Research, 32(1), 182-192.

[18] Hamadene, S., and Morlais, M. A. (2013). Viscosity solutions of systems of PDEs with interconnected obstacles and switching problem. Applied Mathematics \& Optimization, 67(2), 163-196. 
[19] Hamadene, S., Zhang, J. (2010). Switching problem and related system of reflected backward SDEs. Stochastic Processes and their applications, 120(4), 403-426.

[20] Hamadène, S., Zhao, X. (2015). Systems of integro-PDEs with i nterconnected obstacles and multi-modes switching problem driven by Lévy process. Nonlinear Dif. Equat. and Appli. NoDEA, 22(6), 1607-1660.

[21] Harraj, N., Ouknine, Y., Turpin, I. (1900). Double barriers, reflected BSDEs with jumps and viscosity solutions of parabolic integro-differential PDEs. Intern. Jour. of Stochastic Analysis, 2005(1), 37-53.

[22] Hu, Y., Tang, S. (2010). Multi-dimensional BSDE with oblique reflection and optimal switching. Probability Theory and Related Fields, Vol. 147, No. 1-2, pp. 89-121.

[23] Hu, Y., Tang, S. (2013). Switching game of backward stochastic differential equations and associated system of obliquely reflected BSDEs. Discrete and Continuous Dynamical Systems, 35(11), 5447-5465.

[24] H.Ishii, S.Koike (1991). Viscosity Solutions of a System of Nonlinear Second-Order Elliptic PDEs Arising in Switching Games. Funkcialaj Ekvacioj, 34 143-155.

[25] Lenhart, S. M., Yamada, N. (1992). Viscosity solutions associated with switching game for piecewisedeterministic processes. Stochastics: An International Journal of Probability and Stochastic Processes, $38(1), 27-47$.

[26] Pham, H., Vath, V. L., Zhou, X. Y. (2009). Optimal switching over multiple regimes. SIAM Journal on Control and Optimization, 48(4), 2217-2253.

[27] Royer, M. (2006). Backward stochastic differential equations with jumps and related non-linear expectations. Stochastic processes and their applications, 116(10), 1358-1376.

[28] Tang, S., Hou, S. H. (2007). Switching games of stochastic differential systems. SIAM journal on control and optimization, 46(3), 900-929.

[29] Tang, S., Li, X. Necessary conditions for optimal control of stochastic systems with random jumps. SIAM Journal on Control and Optimization, 1994, vol. 32, no 5, p. 1447-1475.

[30] Tang, S., Yong, J. (1993). Finite horizon stochastic optimal switching and impulse controls with a viscosity solution approach. Stochastics: An Intern. Journ. of Proba. and Stoc. Proc., 45(3-4), 145-176.

[31] Zhu, X. (2010). Backward stochastic viability property with jumps and applications to the comparison theorem for multidimensional BSDEs with jumps. AIP Conference Proceedings (Vol.1200, pp.438-441). 


\section{Appendix : Alternative definition of the viscosity solution of system (2.1)}

The following result inspired by the work by Barles-Imbert [4] is another definition of the viscosity solution of system (2.1). We do not give its proof since it is an adaptation of the one given in ([20], Proposition 5.2, pp.1656) as the function $q \longmapsto g^{i j}(t, x, \vec{y}, z, q)$ is non-decreasing, $\beta$ is a bounded function, $\gamma^{i j}$ is non-negative which then imply $I_{i j}(t, x, \phi) \leq I_{i j}(t, x, \psi), I_{i j}^{1, \delta}(t, x, \phi) \leq I_{i j}^{1, \delta}(t, x, \psi)$ and $I_{i j}^{2, \delta}(t, x, \phi) \leq I_{i j}^{2, \delta}(t, x, \psi)$ for any $\phi \leq \psi$ such that $\phi(t, x)=\psi(t, x)=u^{i j}(t, x)(\delta>0$ and $(i, j) \in \Gamma$ are fixed $)$.

Proposition 5.1. A function $\vec{u}=\left(u^{i j}(t, x)\right)_{(i, j) \in \Gamma}:[0, T] \times \mathbf{R}^{k} \rightarrow \mathbf{R}^{m_{1} \times m_{2}}$ such that for any $(i, j) \in \Gamma$, $u^{i j} \in \Pi_{g}$ is lsc (resp. usc) is a viscosity supersolution (resp. subsolution) of (2.1) if:

(i) $v^{i j}\left(T, x_{0}\right) \geq($ resp. $\leq) h^{i j}\left(x_{0}\right), \forall x_{0} \in \mathbf{R}^{k}$;

(ii) For any $\left(t_{0}, x_{0}\right) \in(0, T) \times \mathbf{R}^{k}, \delta \in(0,1)$ and a function $\phi \in \mathcal{C}^{1,2}\left([0, T] \times \mathbf{R}^{k}\right)$ such that $u^{i j}\left(t_{0}, x_{0}\right)=$ $\phi\left(t_{0}, x_{0}\right)$ and $u^{i j}-\phi$ has a global minimum (resp. maximum) at $\left(t_{0}, x_{0}\right)$ on $(0, T) \times B\left(x_{0}, \delta K_{\beta}\right)$ where $K_{\beta}$ is the bound of $\beta$ (see the first inequality of (AO)-(ii)), we have:

$$
\left\{\begin{array}{l}
\min \left\{\left(u^{i j}-L^{i j}[\vec{u}]\right)\left(t_{0}, x_{0}\right) ; \max \left\{\left(u^{i j}-U^{i j}[\vec{u}]\right)\left(t_{0}, x_{0}\right) ;\right.\right. \\
-\partial_{t} \phi\left(t_{0}, x_{0}\right)-b\left(t_{0}, x_{0}\right)^{\top} D_{x} \phi\left(t_{0}, x_{0}\right)-\frac{1}{2} \operatorname{Tr}\left(\sigma \sigma^{\top}\left(t_{0}, x_{0}\right) D_{x x}^{2} \phi\left(t_{0}, x_{0}\right)\right)-I_{\delta}^{1}\left(t_{0}, x_{0}, \phi\right)-I_{\delta}^{2}\left(t_{0}, x_{0}, D_{x} \phi, u^{i j}\right) \\
\left.\left.-g^{i j}\left(t_{0}, x_{0},\left(u^{k l}\left(t_{0}, x_{0}\right)\right)_{(k, l) \in A^{1} \times A^{2}}, \sigma\left(t_{0}, x_{0}\right)^{\top} D_{x} \phi\left(t_{0}, x_{0}\right), I_{i j}^{1, \delta}\left(t_{0}, x_{0}, \phi\right)+I_{i j}^{2, \delta}\left(t_{0}, x_{0}, u^{i j}\right)\right)\right\}\right\} \geq(\text { resp. } \leq) 0 .
\end{array}\right.
$$

Remark 5.1. In taking $\bar{g}_{j l} \equiv+\infty$ (resp. $\left.\underline{g}_{i k} \equiv+\infty\right)$ for any $j, l \in A_{2}$ (resp. $i, k \in A_{1}$ ) we obtain an alternative definition of the viscosity solution of the system of variational inequalities with interconnected lower (resp. upper) obstacles. 